\title{
High energy QCD: Multiplicity distribution and entanglement entropy
}

\author{
E. Gotsman $\oplus^{1, *}$ and E. Levin ${ }^{1,2, \dagger}$ \\ ${ }^{1}$ Department of Particle Physics, School of Physics and Astronomy, Raymond and Beverly Sackler Faculty \\ of Exact Science, Tel Aviv University, Tel Aviv 69978, Israel \\ ${ }^{2}$ Departamento de Física, Universidad Técnica Federico Santa María and Centro Científico-Tecnológico \\ de Valparaíso, Casilla 110-V, Valparaiso, Chile
}

(Received 4 July 2020; accepted 22 September 2020; published 12 October 2020)

\begin{abstract}
In this paper, we show that QCD at high energies leads to the multiplicity distribution $\left(\sigma_{n} / \sigma_{\text {in }}\right)=$ $(1 / N)(N-1 / N)^{n-1}$ (where $N$ denotes the average number of particles) and to entanglement entropy $S=\ln N$, confirming that the partonic state at high energy is maximally entangled. However, the value of $N$ depends on the kinematics of the parton cascade. In particular, for deep inelastic scattering, $N=x G(x, Q)$, where $x G$ is the gluon structure function, while for hadron-hadron collisions, $N \propto Q_{S}^{2}(Y)$, where $Q_{s}$ denotes the saturation scale. We checked that this multiplicity distribution describes the LHC data for low multiplicities $n<(3 \div 5) N$, exceeding it for larger values of $n$. We view this as a consequence of our assumption that the system of partons in hadron-hadron collisions at c.m. rapidity $Y=0$, is dilute. We show that the data can be described at large multiplicities in the parton model, if we do not make this assumption.
\end{abstract}

DOI: $10.1103 /$ PhysRevD.102.074008

\section{INTRODUCTION}

Over the past several years, new ideas have been developed in the high energy and nuclear physics community, which suggest a robust relation between the principle features of high energy scattering and entanglement properties of the hadronic wave function [1-17]. The main idea, which we explore in this paper, is the intimate relation between the entropy in the parton approach [18-21] and the entropy of entanglement in a proton wave function [5].

This relation materialized as the resolution of the following difficulty in our understanding of high energy scattering: on one hand, the proton is a pure state, and it is described by a completely coherent wave function with zero entropy, but, on the other hand, the deep inelastic scattering (DIS) experiments are successfully described, treating the proton as a incoherent collection of quasifree partons. This ensemble has nonvanishing entropy, and Ref. [5] proposes that the origin of this entropy is the entanglement between the degrees of freedom one observes in DIS (partons in the small spatial region of the proton) and the rest of the proton wave function, which is not measured in the DIS experiments.

\footnotetext{
"gotsman@post.tau.ac.il

†leving@tauex.tau.ac.il, eugeny.levin@usm.cl
}

Published by the American Physical Society under the terms of the Creative Commons Attribution 4.0 International license. Further distribution of this work must maintain attribution to the author(s) and the published article's title, journal citation, and DOI. Funded by SCOAP ${ }^{3}$.
In other words, the hadron in the rest frame is described by a pure state $|\psi\rangle$ with density matrix $\hat{\rho}=|\psi\rangle\langle\psi|$ and zero von Neumann entropy $S=-\operatorname{tr}[\hat{\rho} \ln \hat{\rho}]=0$. In DIS at Bjorken $x$ and momentum transfer, $q^{2}=-Q^{2}$ probes only a part of the proton's wave function; let us denote it $A$. In the proton's rest frame, the DIS probes the spatial region $A$ localized within a tube of radius approximately $1 / Q$ and length approximately $1 /(m x)$ [22,23], where $m$ is the proton's mass. The inclusive DIS measurement thus sums over the unobserved part of the wave function localized in the region $B$ complementary to $A$, so we have access only to the reduced density matrix $\hat{\rho}_{A}=\operatorname{tr}_{B} \hat{\rho}$, and not the entire density matrix $\hat{\rho}=|\psi\rangle\langle\psi|$. In Ref. [5], it is proposed that

$$
S_{A}=-\operatorname{tr}_{B}\left[\hat{\rho}_{A} \ln \hat{\rho}_{A}\right]=S_{\text {parton cascade }} .
$$

Equation (1), in spite of its general form, means that we can estimate the entropy and multiplicity distribution of the produced gluons using the parton wave function in the initial state. In addition, we can obtain a thermal distribution of the produced particles in the high energy collision in spite of the fact that the number of secondary interactions in proton-proton collisions is rather low and cannot provide the thermalization due to the interaction in the final state.

It has been demonstrated in Refs. $[6,10,11,16]$ that these ideas are in qualitative and, partly, in quantitative agreement with the available experimental data.

The goal of this paper is to study the multiplicity distribution and the entanglement entropy in the effective theory for QCD at high energies (see Ref. [24] for a general review). Such a theory exists in two different formulations: 
the color glass condensate (CGC)/saturation approach [25-30] and the BFKL (Balitsky-Fadin-Kuraev-Lipatov) Pomeron calculus [31-45].

We believe that the CGC/saturation approach provides a more general pattern $[43,44]$ for the treatment of high energy QCD. However, in this paper, we restrict ourself to the BFKL Pomeron calculus, which has a more direct correspondence with the parton approach and has been used in Ref. [5].

Fortunately, in Ref. [44], it was shown that these two approaches are equivalent for the description of the scattering amplitude

$$
Y \leq \frac{2}{\Delta_{\mathrm{BFKL}}} \ln \left(\frac{1}{\Delta_{\mathrm{BFKL}}^{2}}\right)
$$

where $\Delta_{\mathrm{BFKL}}$ denotes the intercept of the BFKL Pomeron.

The main difference between the CGC approach and the parton QCD cascade for the topics dealt with in this paper is the fact that the CGC approach generates the nondiagonal elements of the density matrix (see, for example, Refs. $[3,9,12,17])$, while for the parton cascade and, generally, in the BFKL Pomeron calculus, the density matrix is diagonal. Since in DIS experiments we can only measure the diagonal elements of density matrix, we can introduce in the framework another kind of entropy: "the entropy of ignorance" [17], which characterized this lack of knowledge of the actual density matrix in DIS experiments. We will show below that the McLerran-Venugopalan approach [25], which is used in Ref. [17], leads to the same multiplicity distribution as the parton cascade.

The paper is organized as follows. In the next section, we consider the entropy and multiplicity distributions in the QCD parton cascade. We show that in different kinematic regions the QCD cascade leads to different energy and dipole size dependence of the mean multiplicity, and that the multiplicity distribution has a general form,

$$
\frac{\sigma_{n}}{\sigma_{\text {in }}}=\frac{1}{N}\left(\frac{N-1}{N}\right)^{n-1},
$$

where $\mathrm{N}$ is the average number of partons. The entanglement entropy is equal to $S_{\text {parton cascade }}=\ln N$, confirming that the partonic state at high energy is maximally entangled [5] (see Fig. 2). In the case of DIS, we argue that $N$ is equal to the gluon structure function, but we can only prove the multiplicity distribution of Eq. (3), for the BFKL evolution of this structure function. In Sec. III, we show that the CGC approach leads to the multiplicity distribution of Eq. (3). In Sec. IV, we consider hadronhadron scattering. In the range of energy given by Eq. (2), we use the Mueller-Patel-Salam-Iancu (MPSI) $[36,46]$ approach, using the formalism of Ref. [41]. We show that in the framework of this approach we have the distribution of Eq. (3), which can describe the experimental data for sufficiently low multiplicities $n \leq(3 \div 5)\langle n\rangle$. However, we fail to describe the data for larger $n$. We conclude that the main assumption of the MPSI approach, that a system of dilute partons are produced in the c.m. rapidity $Y=0$, is not valid for large multiplicities at high energies of the LHC. Unfortunately, at the moment, we have no theoretical tool to treat this scattering. However, in Ref. [47], an approach has been suggested, which allows us to describe the dense system of partons in hadron-hadron collisions, as well as the dilute one. Developing this approach for the multiplicity distribution, we are able to describe the data for large multiplicities. We summarize our results in the conclusions.

\section{QCD PARTON CASCADE}

\section{A. QCD cascade for fast moving large dipole}

\section{General approach}

As discussed in Refs. [24,26,40,41], the parton cascade can be written in the form (see Fig. 1)

$$
\begin{aligned}
& \frac{\partial P_{n}\left(Y, \boldsymbol{r}, \boldsymbol{b} ; \boldsymbol{r}_{1}, \boldsymbol{b}_{1}, \boldsymbol{r}_{2}, \boldsymbol{b}_{2} \ldots \boldsymbol{r}_{i}, \boldsymbol{b}_{i}, \ldots \boldsymbol{r}_{n}, \boldsymbol{b}_{n}\right)}{\partial Y} \\
& =-\sum_{i=1}^{n} \omega_{G}\left(r_{i}\right) P_{n}\left(Y, \boldsymbol{r}, \boldsymbol{b} ; \boldsymbol{r}_{1}, \boldsymbol{b}_{1}, \boldsymbol{r}_{2}, \boldsymbol{b}_{2} \ldots \boldsymbol{r}_{i}, \boldsymbol{b}_{i}, \ldots \boldsymbol{r}_{n}, \boldsymbol{b}_{n}\right) \\
& \quad+\bar{\alpha}_{S} \sum_{i=1}^{n-1} \frac{\left(\boldsymbol{r}_{i}+\boldsymbol{r}_{n}\right)^{2}}{(2 \pi) r_{i}^{2} r_{n}^{2}} P_{n-1}\left(Y, \boldsymbol{r}, \boldsymbol{b} ; \boldsymbol{r}_{1}, \boldsymbol{b}_{1}, \ldots\left(\boldsymbol{r}_{i}+\boldsymbol{r}_{n}\right),\right. \\
& \left.\quad \boldsymbol{b}_{\text {in }}, \ldots \boldsymbol{r}_{n-1}, \boldsymbol{b}_{n}\right),
\end{aligned}
$$

where $P_{n}\left(Y ;\left\{r_{i}, b_{i}\right\}\right)$ is the probability to have $n$-dipoles of size $r_{i}$, at impact parameter $b_{i}$ and at rapidity $Y^{1} \boldsymbol{b}_{\text {in }}$ in Eq. (4) is equal to $\boldsymbol{b}_{\text {in }}=\boldsymbol{b}_{i}+\frac{1}{2} \boldsymbol{r}_{i}=\boldsymbol{b}_{n}-\frac{1}{2} \boldsymbol{r}_{i}$.

Eq. (4) is a typical cascade equation in which the first term describes the reduction of the probability to find $n$ dipoles due to the possibility that one of the $n$ dipoles can decay into two dipoles of arbitrary sizes, while the second term describes the growth due to the splitting of $(n-1)$ dipoles into $n$ dipoles.

The initial condition for the DIS scattering is

$$
\begin{aligned}
P_{1}\left(Y=0, \boldsymbol{r}, \boldsymbol{b} ; \boldsymbol{r}_{1}, \boldsymbol{b}_{1}\right) & =\delta^{(2)}\left(\boldsymbol{r}-\boldsymbol{r}_{1}\right) \delta^{(2)}\left(\boldsymbol{b}-\boldsymbol{b}_{1}\right) ; \\
P_{n>1}\left(Y=0 ;\left\{r_{i}\right\}\right) & =0,
\end{aligned}
$$

which corresponds to the fact that we are discussing a dipole of definite size which develops the parton cascade.

Since $P_{n}\left(Y ;\left\{r_{i}\right\}\right)$ is the probability to find dipoles $\left\{r_{i}\right\}$, we have the sum rule

\footnotetext{
${ }^{1}$ In the laboratory frame rapidity, $Y$ is equal to $Y=$ $y_{\text {dipole } r}-y_{\text {dipoles } r_{i}}$, where $y_{\text {dipole } r}$ is the rapidity of the incoming fast dipole and $y_{\text {dipoles }} r_{i}$ is the rapidity of dipoles $r_{i}$.
} 


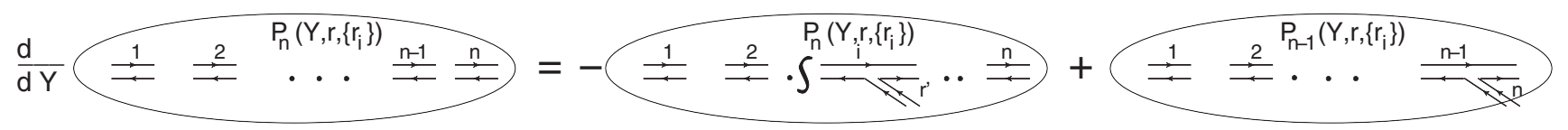

FIG. 1. The graphical form of Eq. (4).

$$
\sum_{n=1}^{\infty} \int \prod_{i=1}^{n} d^{2} r_{i} d^{2} b_{i} P_{n}\left(Y ;\left\{\boldsymbol{r}_{i} \boldsymbol{b}_{i}\right\}\right)=1
$$

i.e., the sum of all probabilities is equal to 1 .

This QCD cascade leads to Balitsky-Kovchegov (BK) equation $[24,27,28]$ for the amplitude and gives the theoretical description of the DIS. We introduce the generating functional [26]

$$
\begin{aligned}
& Z\left(Y, \boldsymbol{r}, \boldsymbol{b} ;\left[u_{i}\right]\right) \\
& \quad=\sum_{n=1}^{\infty} \int P_{n}\left(Y, \boldsymbol{r}, \boldsymbol{b} ;\left\{\boldsymbol{r}_{i} \boldsymbol{b}_{i}\right\}\right) \prod_{i=1}^{n} u\left(\boldsymbol{r}_{i} \boldsymbol{b}_{i}\right) d^{2} r_{i} d^{2} b_{i},
\end{aligned}
$$

where $u\left(\boldsymbol{r}_{i} \boldsymbol{b}_{i}\right) \equiv=u_{i}$ is an arbitrary function. The initial conditions of Eq. (5) and the sum rules of Eq. (6) take the following form for the functional $Z$ :

$$
\begin{gathered}
Z\left(Y=0, \boldsymbol{r}, \boldsymbol{b} ;\left[u_{i}\right]\right)=u(\boldsymbol{r}, \boldsymbol{b}) ; \\
Z\left(Y, r,\left[u_{i}=1\right]\right)=1 .
\end{gathered}
$$

Multiplying both parts of Eq. (4) by $\prod_{i=1}^{n} u\left(\boldsymbol{r}_{i} \boldsymbol{b}_{i}\right)$ and integrating over $r_{i}$ and $b_{i}$ we obtain the following linear functional equation [41]:

$$
\begin{aligned}
& \frac{\partial Z\left(Y, \boldsymbol{r}, \boldsymbol{b} ;\left[u_{i}\right]\right)}{\partial Y} \\
& =\int d^{2} r^{\prime} K\left(\boldsymbol{r}^{\prime}, \boldsymbol{r}-\boldsymbol{r}^{\prime} \mid \boldsymbol{r}\right)(-u(r, b) \\
& \left.\quad+u\left(\boldsymbol{r}^{\prime}, \boldsymbol{b}+\frac{1}{2}\left(\boldsymbol{r}-\boldsymbol{r}^{\prime}\right)\right) u\left(\boldsymbol{r}-\boldsymbol{r}^{\prime}, \boldsymbol{b}-\frac{1}{2} \boldsymbol{r}^{\prime}\right)\right) \frac{\delta Z}{\delta u(r, b)}
\end{aligned}
$$

$$
\begin{aligned}
K\left(\boldsymbol{r}^{\prime}, \boldsymbol{r}-\boldsymbol{r}^{\prime} \mid \boldsymbol{r}\right) & =\frac{\bar{\alpha}_{S}}{2 \pi} \frac{r^{2}}{r^{\prime 2}\left(\boldsymbol{r}-\boldsymbol{r}^{\prime}\right)^{2}} \\
\omega_{G}(r) & =\int d^{2} r^{\prime} K\left(\boldsymbol{r}^{\prime}, \boldsymbol{r}-\boldsymbol{r}^{\prime} \mid \boldsymbol{r}\right) .
\end{aligned}
$$

Searching for the solution of the form $Z\left(\left[u\left(r_{i}, b_{i}, Y\right)\right]\right)$ for the initial conditions of Eq. (8), Eq. (9a) can be rewritten as the nonlinear equation [26]:

$$
\begin{aligned}
& \frac{\partial Z\left(Y, \boldsymbol{r}, \boldsymbol{b} ;\left[u_{i}\right]\right)}{\partial Y} \\
& =\int d^{2} r^{\prime} K\left(\boldsymbol{r}^{\prime}, \boldsymbol{r}-\boldsymbol{r}^{\prime} \mid \boldsymbol{r}\right)\left\{Z\left(r^{\prime}, \boldsymbol{b}+\frac{1}{2}\left(\boldsymbol{r}-\boldsymbol{r}^{\prime}\right) ;\left[u_{i}\right]\right)\right. \\
& \left.\quad \times Z\left(\boldsymbol{r}-\boldsymbol{r}^{\prime}, \boldsymbol{b}-\frac{1}{2} \boldsymbol{r}^{\prime} ;\left[u_{i}\right]\right)-Z\left(Y, \boldsymbol{r}, \boldsymbol{b} ;\left[u_{i}\right]\right)\right\}
\end{aligned}
$$

Therefore, the QCD parton cascade of Eq. (4) takes into account nonlinear evolution. However, to obtain the BK equation for the scattering amplitude, we need to introduce the scattering amplitude $\gamma\left(r_{i}, b\right)$, for the interaction of the dipole with the target at low energies. Using these amplitudes, we can obtain the nonlinear BK equation from Eq. (10), since [28]

$$
\begin{aligned}
N(Y, r, b)= & \sum_{n=1}^{\infty} \frac{(-1)^{n-1}}{n !} \int \prod_{i=1}^{n}\left(d^{2} r_{i} \gamma\left(r_{i}, b\right) \frac{\delta}{\delta u_{i}}\right) \\
& \times\left. Z\left(Y, r, b,\left[u_{i}\right]\right)\right|_{u_{i}=1} .
\end{aligned}
$$

Using Eqs. (9a) and (11), we derive the BK equation in the standard form:

$$
\begin{aligned}
\frac{\partial}{\partial Y} N(\boldsymbol{r}, \boldsymbol{b}, Y)= & \int d^{2} \boldsymbol{r}^{\prime} K\left(\boldsymbol{r}^{\prime}, \boldsymbol{r}-\boldsymbol{r}^{\prime} \mid \boldsymbol{r}\right)\left\{N\left(\boldsymbol{r}^{\prime}, \boldsymbol{b}-\frac{1}{2}\left(\boldsymbol{r}-\boldsymbol{r}^{\prime}\right), Y\right)\right. \\
& +N\left(\boldsymbol{r}-\boldsymbol{r}^{\prime}, \boldsymbol{b}-\frac{1}{2} \boldsymbol{r}^{\prime}, Y\right)-N(\boldsymbol{r}, \boldsymbol{b}, Y) \\
& \left.-N\left(\boldsymbol{r}-\boldsymbol{r}^{\prime}, \boldsymbol{b}-\frac{1}{2} \boldsymbol{r}^{\prime}, Y\right) N\left(\boldsymbol{r}^{\prime}, \boldsymbol{b}-\frac{1}{2}\left(\boldsymbol{r}-\boldsymbol{r}^{\prime}\right), Y\right)\right\} .
\end{aligned}
$$

\section{Several first iterations}

Our goal is to find the solution to Eq. (4). In particular, for the multiplicity distribution and for the entropy, we wish to find

$$
\tilde{P}_{n}(Y, r)=\int P_{n}\left(Y, \boldsymbol{r}, \boldsymbol{b},\left\{\boldsymbol{r}_{i}, \boldsymbol{b}^{\prime}\right) \prod_{n=1}^{n} d^{2} r_{i} d^{2} b^{\prime}\right.
$$

$\tilde{P}_{n}$ is the probability of finding $n$ dipoles of all possible sizes at the same values of the impact parameters, and being such, it gives $\sigma_{n} / \sigma_{\text {in }}$, which is the multiplicity distribution in the QCD parton cascade. The initial and boundary conditions for $\tilde{P}_{n}(Y, r)$ follow from Eq. (5) and Eq. (6) and take the form: 
$\tilde{P}_{1}(Y=0, r, b)=1 ; \quad \tilde{P}_{n}(Y=0, r)=0 \quad$ for $n>1 ;$

$\sum_{n=1}^{\infty} \tilde{P}_{1}(Y, r, b)=1$.

First, let us find $\tilde{P}_{1}(Y, r)$. The equation for $P_{1}$ has the form

$$
\frac{\partial P_{1}\left(Y, r, b, r_{1}, b_{1}\right)}{\partial Y}=-\omega_{G}\left(r_{1}\right) P_{1}\left(Y, r, b, r_{1}, b_{1}\right)
$$

with the initial condition

$$
P_{1}\left(Y=0, r, b, r_{1}, b_{1}\right)=\delta^{(2)}\left(\boldsymbol{r}-\boldsymbol{r}_{1}\right) \delta^{(2)}\left(\boldsymbol{b}-\boldsymbol{b}_{1}\right)
$$

Therefore, for $\tilde{P}_{1}(Y, r, b)$, the equation takes the form

$$
\frac{\partial \tilde{P}_{1}(Y, r, b)}{\partial Y}=-\omega_{G}(r) \tilde{P}_{1}(Y, r, b)
$$

with the solution

$$
\tilde{P}_{1}(Y, r, b)=e^{-\omega_{G}(r) Y}
$$

The equation for $P_{2}\left(Y, r, b, r_{1}, b^{\prime}, r_{2}, b^{\prime}\right)$ has the following form:

$$
\begin{gathered}
\frac{\partial P_{2}\left(Y, r, b ; r_{1}, b^{\prime}, r_{2}, b^{\prime}\right)}{\partial Y} \\
=-\left(\omega_{G}\left(r_{1}\right)+\omega_{G}\left(r_{2}\right)\right) P_{2}\left(Y, r, b, r_{1}, b^{\prime}, r_{2}, b\right) \\
\quad+\frac{\bar{\alpha}_{S}}{2 \pi} \frac{\left(\boldsymbol{r}_{1}+\boldsymbol{r}_{2}\right)^{2}}{r_{1}^{2} r_{2}^{2}} P_{1}\left(Y, r, b ; \boldsymbol{r}_{1}+\boldsymbol{r}_{2}, b^{\prime}\right) .
\end{gathered}
$$

First, let us estimate the value of $\omega_{G}(r)$, which is given by Eq. (9b):

$$
\begin{aligned}
& \omega_{G}(r)=\frac{\bar{\alpha}_{S}}{2 \pi} \int d^{2} r^{\prime} \frac{r^{2}}{r^{\prime 2}\left(\boldsymbol{r}-\boldsymbol{r}^{\prime}\right)^{2}} \\
& =\frac{\bar{\alpha}_{S}}{\pi} \int d^{2} r^{\prime} \frac{r^{2}}{r^{\prime 2}\left(r^{\prime 2}+\left(\boldsymbol{r}-\boldsymbol{r}^{\prime}\right)^{2}\right)} \\
& =\left\{\int_{r_{0}}^{r}+\int_{r}^{\infty}\right\} d^{2} r^{\prime} \frac{r^{2}}{r^{\prime 2}\left(r^{\prime 2}+\left(\boldsymbol{r}-\boldsymbol{r}^{\prime}\right)^{2}\right)} \\
& =\bar{\alpha}_{S} \underbrace{\ln \left(r^{2} / r_{0}^{2}\right)}_{r^{\prime} \leq r}+\underbrace{0}_{r^{\prime} \geq r}=\bar{\alpha}_{S} \int_{r_{0}^{2}}^{r^{2}} \frac{d r^{\prime 2}}{r^{\prime 2}} \text {. }
\end{aligned}
$$

Hence, only dipoles of size smaller than $r$ contribute to the value of $\omega_{G}(r)$.
We suggest that the solution to Eq. (19) has the form

$$
\begin{aligned}
& \int d^{2} b^{\prime} P_{2}\left(Y, r, b ; r_{1}, b^{\prime}, r_{2}, b^{\prime}\right) \\
& =\frac{1}{r_{1}^{2} r_{2}^{2}} \Theta\left(r-r_{1}\right) \Theta\left(r-r_{2}\right) p_{2}(r, b),
\end{aligned}
$$

where $\Theta(z)$ denotes the step function: $\Theta(z)=1$ for $z>0$ and $\Theta(z)=0$ for $z<0$. For the solution of Eq. (21), we can obtain the equation for $\tilde{P}_{2}(Y, r, b)$, integrating both parts of Eq. (19) over $b^{\prime}, r_{1}$, and $r_{2}$. It has the form

$$
\frac{\partial \tilde{P}_{2}(Y, r)}{\partial Y}=-2 \omega_{G}(r) \tilde{P}_{2}(Y, r)+\omega_{G}(r) \tilde{P}_{1}(Y, r)
$$

Using Eq. (18), we obtain that

$$
\tilde{P}_{2}(Y, r)=e^{-\omega_{G}(r) Y}\left(1-e^{-\omega_{G}(r) Y}\right)
$$

One can see that Eq. (23) gives $\tilde{P}_{2}(Y=0, r)=0$ in accord with Eq. (14). For small $\omega_{G}(r) Y \ll 1$ in the parton cascade, only two terms exist, $\tilde{P}_{1}(Y, r)$ and $\tilde{P}_{2}(Y, r)$, and Eq. (6) reduces to

$\tilde{P}_{1}(Y, r)+\tilde{P}_{2}(Y, r) \stackrel{\omega_{G}(r) Y \ll 1}{\longrightarrow} \underbrace{1-\omega_{G}(r) Y}_{\tilde{P}_{1}}+\underbrace{\omega_{G}(r) Y}_{\tilde{P}_{2}}=1$.

Eq. (24) shows that $P_{n}\left(Y, r, b,\left\{r_{i}, b^{\prime}\right\}\right)$ are negligibly small for dipoles with large sizes $\left(r_{i}>r\right)$.

\section{Solution}

We suggest looking for the general solution in the form

$$
\tilde{P}_{n}\left(Y, \boldsymbol{r}, \boldsymbol{b} ;\left\{\boldsymbol{r}_{i}^{\prime}\right\}\right)=\prod_{i=1}^{n} \Theta\left(r-r_{i}\right) \frac{1}{r_{i}^{2}} \tilde{P}_{n}(Y, r ; b) .
$$

For such a solution, we can obtain from Eq. (4) the following equations for $\tilde{P}_{n}(Y, r ; b)$ :

$$
\begin{aligned}
\frac{\partial \tilde{P}_{n}(Y, r ; b)}{\partial Y}= & -n \omega_{G}(r) \tilde{P}_{n}(Y, r ; b) \\
& +(n-1) \omega_{G}(r) \tilde{P}_{n-1}(Y, r ; b) .
\end{aligned}
$$

Introducing the Laplace transform

$$
\tilde{P}_{n}(Y, r ; b)=\int_{\epsilon-i \infty}^{\epsilon+i \infty} \frac{d \omega}{2 \pi i} e^{\omega Y} \tilde{p}_{n}(\omega, r ; b),
$$

we rewrite Eq. (26) in the form

$$
\begin{aligned}
\omega \tilde{p}_{n}(\omega, r ; b)= & -n \omega_{G}(r) \tilde{p}_{n}(\omega, r ; b) \\
& +(n-1) \omega_{G}(r) \tilde{p}_{n-1}(\omega, r ; b) .
\end{aligned}
$$


Eq. (28) has the solution

$$
\tilde{p}_{n}(\omega, r)=(n-1) ! \prod_{m=1}^{n} \frac{1}{\omega+m \omega_{G}(r)} ;
$$

note that Eq. (29) reproduces both Eq. (18) and Eq. (23).

Taking the inverse Laplace transform of function $e^{-\omega_{G}(r) Y}\left(1-e^{-\omega_{G}(r) Y}\right)^{n-1}$, we have

$$
\begin{aligned}
& \int_{0}^{\infty} d Y e^{-\omega Y} e^{-\omega_{G}(r) Y}\left(1-e^{-\omega_{G}(r) Y}\right)^{n-1} \\
& \quad=(n-1) ! \prod_{m=1}^{n} \frac{\omega_{G}(r)}{\omega+m \omega_{G}(r)}=\tilde{p}_{n}(\omega, r) .
\end{aligned}
$$

Hence, we have the following solution:

$$
\tilde{P}_{n}(Y, r)=e^{-\omega_{G}(r) Y}\left(1-e^{-\omega_{G}(r) Y}\right)^{n-1} .
$$

It is easy to see that Eq. (31) satisfies the initial conditions and the sum rules of Eq. (14).

\section{B. Multiplicity distribution and entropy of the parton cascade}

As has been mentioned,

$$
\frac{\sigma_{n}}{\sigma_{\text {in }}}=\tilde{P}_{n}(Y, r)
$$

and therefore determines the multiplicity distribution. Calculating the average $N$,

$$
N=\sum_{n=1}^{\infty} n \frac{\sigma_{n}}{\sigma_{\text {in }}}=\sum_{n=1}^{\infty} n \tilde{P}_{n}(Y, r)=e^{\omega(r) Y}
$$

we see that this distribution can be written in the form

$$
\frac{\sigma_{n}}{\sigma_{\text {in }}}=\frac{1}{N}\left(\frac{N-1}{N}\right)^{n-1}=\frac{1}{\bar{N}}\left(\frac{\bar{N}}{\bar{N}+1}\right)^{n},
$$

where we have denoted $\bar{N}=N-1$.

We can compare Eq. (34) with the general form of the negative binomial distribution (NBD)

$$
P^{\mathrm{NBD}}(r, \bar{n}, n)=\left(\frac{r}{r+\langle n\rangle}\right)^{r} \frac{\Gamma(n+r)}{n ! \Gamma(r)}\left(\frac{\langle n\rangle}{r+\langle n\rangle}\right)^{n} .
$$

One can see that Eq. (34) can be rewritten as

$$
\frac{\sigma_{n}}{\sigma_{\text {in }}}=\frac{\bar{N}}{\bar{N}+1} P^{\mathrm{NBD}}(1, \bar{N}, n),
$$

where $\sigma_{n}$ is the cross section for producing $n$ hadrons in a collision and $\sigma_{\text {in }}$ is the inelastic cross section. Therefore, at large $\bar{N}$, our distribution is close to the negative binomial distribution with number of failures $r=1$ and with probability of success $p=\bar{N} /(\bar{N}+1)$. Eq. (34) coincides with the multiplicity distribution in the parton model (see below and Ref. [5]). The difference is only in the expression for the average multiplicity $(N)$.

Having Eq. (34), we can calculate the von Neumann entropy of the parton cascade [see Eq. (1)], given by the Gibbs formula,

$$
S_{\text {parton cascade }}=-\sum_{n} p_{n} \ln p_{n},
$$

where $p_{n}$ is the probabilities of microstates. In the parton cascade, we can identify $p_{n}$ with $\tilde{P}_{n}(Y, r)$, reducing Eq. (38) to the following expression (see Fig. 2):

$$
\begin{aligned}
S_{\text {parton cascade }} & =-\sum_{n} \tilde{P}_{n}(Y, r) \ln \tilde{P}_{n}(Y, r) \\
& =\sum_{n}\left(\ln \bar{N}-n \ln \left(\frac{\bar{N}}{\bar{N}+1}\right)\right) \frac{1}{\bar{N}}\left(\frac{\bar{N}}{\bar{N}+1}\right)^{n} \\
& =\ln \bar{N}+\ln \left(\frac{\bar{N}}{\bar{N}+1}\right)\left(1+\frac{1}{\bar{N}}\right) \stackrel{\bar{N} \gg 1}{\longrightarrow} \ln (N-1) \\
& =\omega_{G}(r) Y .
\end{aligned}
$$

Eq. (38) shows that at large $Y$ all probabilities $\tilde{P}_{n}$ become equal and small, of the order of $\tilde{P}_{n} \sim \frac{1}{N}$. It is well known that this equipartitioning of microstates maximizes the von Neumann entropy and describes the maximally entangled state. We thus conclude that at large $Y$ the fast dipole represents a maximally entangled quantum state of partons.

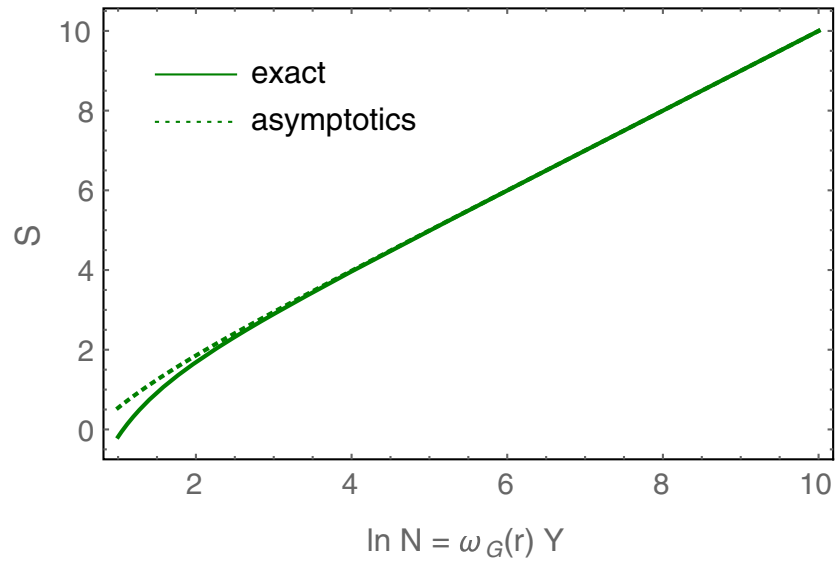

FIG. 2. Entropy $S$ vs $\ln N=\omega_{G}(r) Y$ [see Eq. (38)]. $S_{\text {asymp }}=$ $\ln N=\omega_{G}(r) Y$. 


\section{QCD motivated parton model}

The main assumption of the parton model [19-21] is that all partons have average transverse momentum, which does not depend on energy. Therefore, we can obtain the parton model from the QCD cascade assuming that the unknown confinement of gluons leads to the QCD cascade for the dipole of fixed size. In this case, the cascade equation take the form

$$
\frac{d P_{n}(Y)}{d Y}=-\Delta n P_{n}(Y)+(n-1) \Delta P_{n-1}(Y),
$$

where $P_{n}(Y)$ is the probability to find $n$ dipoles (of a fixed size in our model) at rapidity $Y$ and $\Delta=\omega_{G}\left(r=r_{0}\right)$.

Using the Laplace transform of Eq. (27), we obtain the solution to Eq. (39) in the form

$$
P_{n}(\omega)=(n-1) ! \prod_{m=1}^{n} \frac{1}{\omega+m \Delta} .
$$

Using Eq. (30), we see that the solution of Eq. (39) has the form

$\frac{\sigma_{n}}{\sigma_{\text {in }}} \equiv P_{n}(Y)=e^{-\Delta Y}\left(1-e^{-\Delta Y}\right)^{n-1}=\frac{1}{N}\left(\frac{N-1}{N}\right)^{n-1}$,

which is a direct generalization of Eq. (31). In Eq. (41), $N$ is the average number of the partons, which is equal to $N=\exp (\Delta Y)$. In the parton model, the average number of partons is related to the deep inelastic structure function. Therefore, in Ref. [5], it is assumed that

$$
N=x G\left(x, Q^{2}\right),
$$

where $x G$ is the gluon structure function. In this case, $\Delta$ can be identified with the intercept of the BFKL Pomeron [31]. One can see that $N=\exp \left(\omega_{G}(r) Y\right)$; this is certainly not the same as a solution of the QCD evolution for the gluon structure function.

\section{Mutiplicity distribution for the parton cascade in DIS}

As we have seen [see Eq. (25)], our solution describes the evolution in the system of partons with smaller sizes of dipoles than the initial fastest dipole. On the other side, only partons of larger than initial parton size contribute to the structure function. In double log approximation, the emission of such dipoles leads to $x G \propto \exp \left(2 \sqrt{\bar{\alpha}_{S} Y \ln \left(R^{2} / r^{2}\right)}\right)$, where $R$ is the size of the target. We need to consider how the produced dipoles interact with the target. We measure the gluon structure function in the experiment in which $r^{2}$ of the fastest dipole is about $r^{2} \sim 1 / Q^{2} \ll R^{2}$. Since all $r_{i} \leq r$ the $n$ produced dipoles interact with the target at rapidity $Y$ and since $r_{i}<R$, the amplitude of this interaction is proportional to $r_{i}^{2} / R^{2}$. This fact completely changes the structure of the cascade. Let us illustrate this, considering $P_{2}\left(Y, r ; r_{1}, r_{2}\right)$. The amplitude of interaction is proportional to $\quad \rho_{2}\left(Y, r, b ; r_{1}, r_{2}, b^{\prime}\right) \equiv\left(r_{1}^{2}+r_{2}^{2}\right) P_{2}\left(Y, r, b ; r_{1}, r_{2}, b^{\prime}\right)$. Let us look at Eq. (19) for $r_{i}>r$. The term with gluon $\omega_{G}\left(r_{i}\right)$ does not contribute since, as we have discussed, only $r_{i}>r$ contribute in this term. Therefore, the equation reduces to the following one:

$$
\begin{aligned}
\frac{\partial \rho_{2}\left(Y, r, b ; r_{1}, r_{2}, b^{\prime}\right)}{\partial Y} & =\frac{r^{2}}{r_{1}^{4}} 2 r_{1}^{2} P\left(Y, r, b ; r_{1} \cdot b^{\prime}\right) \\
& =2 \frac{1}{r_{1}^{2}} P\left(Y, r, b ; r_{1} \cdot b^{\prime}\right) .
\end{aligned}
$$

Therefore, we infer that dipole sizes larger than $r$ contribute to the scattering amplitude of our interest and lead to large $\rho_{2}$.

Generally speaking, the scattering amplitude can be written in the form $[28,41]$

$$
\begin{aligned}
N(Y, r, b)= & -\sum_{n=1}^{\infty}(-1)^{n} \rho_{n}^{p}\left(r_{1}, b_{1}, \ldots r_{n}, b_{n} ; Y-Y_{0}\right) \\
& \times \prod_{i=1}^{n} N\left(Y_{0}, r_{i}, b_{i}\right) d^{2} r_{i} d^{2} b_{i},
\end{aligned}
$$

where $N\left(Y_{0}, r_{i}, b_{i}\right)$ is the amplitude of the interaction of dipole $r_{i}$ with the target at low energy $Y=Y_{0}$ and the $n$-dipole densities in the projectile $\rho_{n}^{p}\left(r_{1}, b_{1}, \ldots, r_{n}, b_{n}\right)$ are defined as follows:

$\rho_{n}^{p}\left(r_{1}, b_{1} \ldots, r_{n}, b_{n} ; Y-Y_{0}\right)=\left.\frac{1}{n !} \prod_{i=1}^{n} \frac{\delta}{\delta u_{i}} Z\left(Y-Y_{0} ;[u]\right)\right|_{u=1}$.

For $\rho_{n}$, we obtain [41]

$$
\begin{aligned}
\frac{\partial \rho_{n}^{p}\left(r_{1}, b_{1} \ldots, r_{n}, b_{n}\right)}{\bar{\alpha}_{s} \partial Y} \\
=-\sum_{i=1}^{n} \omega\left(r_{i}\right) \rho_{n}^{p}\left(r_{1}, b_{1} \ldots, r_{n}, b_{n}\right) \\
\quad+2 \sum_{i=1}^{n} \int \frac{d^{2} r^{\prime}}{2 \pi} \frac{r^{\prime 2}}{r_{i}^{2}\left(\boldsymbol{r}_{i}-\boldsymbol{r}^{\prime}\right)^{2}} \rho_{n}^{p}\left(\ldots r^{\prime}, b_{i}-r^{\prime} / 2 \ldots\right) \\
\quad+\sum_{i=1}^{n-1} \frac{\left(\boldsymbol{r}_{i}+\boldsymbol{r}_{n}\right)^{2}}{(2 \pi) r_{i}^{2} r_{n}^{2}} \rho_{n-1}^{p}\left(\ldots\left(\boldsymbol{r}_{i}+\boldsymbol{r}_{n}\right), b_{\mathrm{in}} \ldots\right) .
\end{aligned}
$$

For $\rho_{1}$, we have the linear equation: 


$$
\begin{aligned}
\frac{\partial \rho_{1}^{p}\left(Y ; r_{1}, b\right)}{\bar{\alpha}_{S} \partial Y}= & -\omega_{G}\left(r_{1}\right) \rho_{1}^{p}\left(Y ; r_{1}, b\right) \\
& +2 \int \frac{d^{2} r^{\prime}}{2 \pi} \frac{r^{\prime 2}}{r_{1}^{2}\left(\boldsymbol{r}_{1}-\boldsymbol{r}^{\prime}\right)^{2}} \bar{\rho}_{1}^{p}\left(Y, r^{\prime}, b\right) .
\end{aligned}
$$

Introducing $\bar{\rho}_{1}^{p}\left(Y ; r_{1}, b\right)=r_{1}^{2} \rho_{1}^{p}\left(Y, r^{\prime}, b\right)$, we obtain for $\bar{\rho}_{1}^{p}\left(Y r_{1}, b\right)$ the BFKL equation:

$$
\begin{aligned}
\frac{\partial \bar{\rho}_{1}^{p}\left(Y ; r_{1}, b\right)}{\bar{\alpha}_{S} \partial Y}= & -\omega_{G}\left(r_{1}\right) \bar{\rho}_{1}^{p}\left(Y ; r_{1}, b\right) \\
& +2 \int \frac{d^{2} r^{\prime}}{2 \pi} \frac{1}{\left(\boldsymbol{r}_{1}-\boldsymbol{r}^{\prime}\right)^{2}} \bar{\rho}_{1}^{p}\left(Y, r^{\prime}, b\right) .
\end{aligned}
$$

The physical meaning of $\rho_{1}^{p}$ is clear from Eq. (45); it is the mean number of dipoles with size $r_{1}$ that have been produced. The multiplicity, which we needed in DIS, is the number of dipoles with sizes larger than $r \sim 1 / Q$. It is equal to

$$
\begin{aligned}
N_{1}^{p}(Y, r) & =\int_{r} d^{2} r_{1}, d^{2} b \rho_{1}^{p}\left(Y ; r_{1}, b\right) \\
& =\int^{\xi} d \xi^{\prime} d^{2} b \bar{\rho}_{1}^{p}\left(Y, \xi^{\prime}, b\right)=\langle n\rangle,
\end{aligned}
$$

where $\xi=\ln \left(1 / r^{2}\right)$.

In the double log approximation (DLA) of perturbative QCD, Eq. (48) can be rewritten in the form

$$
\frac{\partial^{2} N_{1}^{p}(Y, r)}{\bar{\alpha}_{S} \partial Y \partial \xi}=N_{1}^{p}(Y, r)
$$

It is worth it to mention that $N_{1}^{p}$ is the gluon structure function in the DLA.

The equation for $\bar{\rho}_{2}^{p}\left(Y ; r_{1}, r_{2}, b\right)$ has the form ${ }^{2}$

$$
\begin{aligned}
\frac{\partial \rho_{2}^{p}\left(Y ; r_{1}, r_{2}, b\right)}{\bar{\alpha}_{S} \partial Y}= & -\left(\omega_{G}\left(r_{1}\right)+\omega_{G}\left(r_{2}\right)\right) \rho_{2}^{p}\left(Y ; r_{1}, r_{2} b\right) \\
& +2 \int \frac{d^{2} r^{\prime}}{2 \pi} \frac{r^{\prime 2}}{r_{1}^{2}\left(\boldsymbol{r}_{1}-\boldsymbol{r}^{\prime}\right)^{2}} \rho_{2}^{p}\left(Y, r^{\prime}, b, r_{2}, b\right) \\
& +2 \int \frac{d^{2} r^{\prime}}{2 \pi} \frac{r^{\prime 2}}{r_{2}^{2}\left(\boldsymbol{r}_{2}-\boldsymbol{r}^{\prime}\right)^{2}} \rho_{2}^{p}\left(Y, r_{1}, r^{\prime}, b\right) \\
& \left.+\frac{\left(\boldsymbol{r}_{1}+\boldsymbol{r}_{2}\right)^{2}}{(2 \pi) r_{1}^{2} r_{2}^{2}} \rho_{1}^{p}\left(Y ; \boldsymbol{r}_{1}+\boldsymbol{r}_{2}\right), b\right)
\end{aligned}
$$

However, to find the multiplicity distribution, we need to introduce moments [see Eq. (13)]:

\footnotetext{
${ }^{2}$ For simplicity of presentation, we took $b \gg r_{i}$.
}

$$
\begin{aligned}
N_{n}^{p}(Y, r) & =\int \prod_{i=1}^{n} d^{2} r_{i} d^{2} b \rho_{n}^{p}\left(Y,\left\{r_{i}\right\}, b\right) \\
& =\int \prod_{i=1}^{n} \frac{d^{2} r_{i}}{r_{i}^{2}} d^{2} b \bar{\rho}_{n}^{p}\left(Y,\left\{r_{i}\right\}, b\right)
\end{aligned}
$$

$N_{2}^{p}$ is equal to

$N_{2}^{p}(Y, r)=\int^{\xi} d \xi_{1} \int^{\xi} d \xi_{2} \int d^{2} b \bar{\rho}_{2}^{p}\left(Y, \xi_{1}, \xi_{2}, b\right)$,

which gives $\left\langle\frac{n(n-1)}{2}\right\rangle$.

The equation for $\rho_{2}^{p}$ can be rewritten in the form for $\bar{\rho}_{2}^{p}$

$$
\begin{aligned}
\frac{\partial \bar{\rho}_{2}^{p}\left(Y ; r_{1}, r_{2}, b\right)}{\bar{\alpha}_{S} \partial Y}= & -\left(\omega_{G}\left(r_{1}\right)+\omega_{G}\left(r_{2}\right)\right) \bar{\rho}_{2}^{p}\left(Y ; r_{1}, r_{2} b\right) \\
& +2 \int \frac{d^{2} r^{\prime}}{2 \pi} \frac{1}{\left(\boldsymbol{r}_{1}-\boldsymbol{r}^{\prime}\right)^{2}} \bar{\rho}_{2}^{p}\left(Y, r^{\prime}, b, r_{2}, b\right) \\
& +2 \int \frac{d^{2} r^{\prime}}{2 \pi} \frac{1}{\left(\boldsymbol{r}_{2}-\boldsymbol{r}^{\prime}\right)^{2}} \bar{\rho}_{2}^{p}\left(Y, r_{1}, r^{\prime}, b\right) \\
& +\bar{\rho}_{1}^{p}\left(Y ; \boldsymbol{r}_{1}+\boldsymbol{r}_{2}, b\right),
\end{aligned}
$$

or in DLA, it takes the form

$$
\begin{aligned}
\frac{\partial \bar{\rho}_{2}^{p}\left(Y ; \xi_{1}, \xi_{2}, b\right)}{\bar{\alpha}_{S} \partial Y}= & \int \xi^{\xi_{1}} d \xi^{\prime} \bar{\rho}_{2}^{p}\left(Y, \xi^{\prime}, \xi_{2}, b\right) \\
& +\int^{\xi_{2}} d \xi^{\prime} \bar{\rho}_{2}^{p}\left(Y, \xi_{1}, \xi^{\prime}, b\right) \\
& +\bar{\rho}_{1}^{p}\left(\xi_{1} \approx \xi_{2}\right)
\end{aligned}
$$

Note that the gluon Reggeization does not contribute in DLA, since it describes the contribution of distances $r_{i}<r$ (see the discussion above). Integrating Eq. (55) over $\xi_{1}$ and $\xi_{2}$, we obtain

$$
\frac{\partial N_{2}^{p}(Y, \xi)}{\bar{\alpha}_{S} \partial Y}=2 \int^{\xi} d \xi^{\prime} N_{2}^{p}\left(Y, \xi^{\prime}\right)+\int^{\xi} d \xi^{\prime} N_{1}^{p}\left(Y, \xi^{\prime}\right) .
$$

The general solution to Eq. (56) has a form $N_{2}^{p}(Y, \xi)=$ $N_{2}^{p, \text { homog }}(Y, \xi)-N_{1}^{p}(Y, \xi)$, where $N_{2}^{p, \text { homog }}(Y, \xi)$ is the solution of the homogenous equation:

$$
\frac{\partial N_{2}^{p, \operatorname{homog}}(Y, \xi)}{\bar{\alpha}_{S} \partial Y}=2 \int^{\xi} d \xi^{\prime} N_{2}^{p, \text { homog }}(Y, \xi) .
$$

The solution of Eq. (57) has the form

$$
N_{2}^{p, \operatorname{homog}}(Y, \xi)=\int_{\epsilon-i \infty}^{\epsilon+i \infty} \frac{d \gamma}{2 \pi i} e^{\frac{2 \bar{\alpha}}{\gamma} Y+\gamma \xi} n_{\text {in }}(\gamma),
$$

where $\omega(\gamma)=\frac{\bar{\alpha}_{S}}{\gamma}$ is the DLA limit of the BFKL kernel, 


$$
\omega(\gamma)=\bar{\alpha}_{S} \chi(\gamma)=\bar{\alpha}_{S}(2 \psi(1)-\psi(\gamma)-\psi(1-\gamma)),
$$

where $\psi(z)$ is Euler gamma function [see Ref. [48], Eq. (8.36)].

We select $n_{\text {in }}(\gamma)=1 / \gamma$, since at $Y=0$ we have only one dipole and $N_{2}=\langle n(n-1) / 2\rangle=0$. Taking the integral over $\gamma$ using the method of steepest descent, we obtain

$$
N_{2}^{p, \text { homog }}(Y, \xi)=\left(\frac{\pi}{2 \sqrt{2 \bar{\alpha}_{S} Y \xi}}\right)^{1 / 2} \exp \left(2 \sqrt{2 \bar{\alpha}_{S} Y \xi}\right) .
$$

First, we wish to note that Eq. (60) leads to $N_{2}^{p \text {,homog }}(Y, \xi) \neq\left(N_{1}^{p}(Y, \xi)\right)^{2}$. However, in the diffusion approximation for the BFKL kernel,

$$
\begin{aligned}
\omega(\gamma) & =\Delta_{\mathrm{BFKL}}+D(1 / 2-\gamma)^{2} \\
& =\Delta_{\mathrm{BFKL}}-D \nu^{2} \quad \text { with } \quad \gamma=\frac{1}{2}+i \nu, \\
\Delta_{\mathrm{BFKL}} & =4 \ln 2 \bar{\alpha}_{S} ; D=14 \zeta(3) \bar{\alpha}_{S}=16.828 \bar{\alpha}_{S},
\end{aligned}
$$

the main contribution stems from $\omega=\Delta_{\mathrm{BFKL}}$ and $N_{2}^{p \text {,homog }}(Y, \xi)=\left(N_{1}^{p}(Y, \xi)\right)^{2}$, if we neglect the contributions at $\gamma \neq 1 / 2 \nu \neq 0$ ). Taking the integral over $\gamma$ using the method of steepest descent for the kernel of Eq. (61), one can see that the values of the saddle point for $\nu$ are equal,

$$
\nu_{\mathrm{SP}}=\frac{\xi}{2 D n Y},
$$

for $N_{n}^{P}$. Therefore, for large $Y$ as well as for large $n$, we, indeed, can consider $\nu_{\mathrm{SP}} \rightarrow 0$. For this special case, we have

$$
N_{2}^{p}(Y, \xi)=\left(N_{1}^{p}(Y, \xi)\right)^{2}-N_{1}^{p}(Y, \xi) .
$$

Comparing with the multiplicity distribution of Eq. (34), one can see that Eq. (57) gives the factorial moment $\left\langle\frac{n(n-1)}{2}\right\rangle$ of this distribution with $\langle n\rangle=N_{1}^{p}$.

For $N_{n}^{p}$, the equation follows from Eq. (53), which in DLA takes the form

$$
\begin{aligned}
\frac{\partial N_{n}^{p}(Y, \xi)}{\bar{\alpha}_{S} \partial Y}= & n \int^{\xi} d \xi^{\prime} N_{n}^{p}\left(Y, \xi^{\prime}\right) \\
& +(n-1) \int^{\xi} d \xi^{\prime} N_{n-1}^{p}\left(Y, \xi^{\prime}\right)
\end{aligned}
$$

The solution to this equation has the form

$N_{n}^{p}(Y, \xi)=\int_{\epsilon-i \infty}^{\epsilon+i \infty} \frac{d \gamma}{2 \pi i} \frac{1}{\gamma} e^{\omega(\gamma) Y+\gamma \xi}\left\{e^{\omega(\gamma) Y}-1\right\}^{n-1}$,

with $\omega(\gamma)=\bar{\alpha}_{S} / \gamma$ in DLA.

Comparing Eq. (65) with the moments $M_{q}=\left\langle\frac{n !}{q !(n-q) !}\right\rangle$ for the multiplicity distribution of Eq. (34),
$N_{1}=\bar{n} ; \quad N_{k}(k>1)=\left\langle\frac{n !}{q !(n-q) !}\right\rangle=\bar{n}(\bar{n}-1)^{k-1}$,

one can see that $N_{n}^{p}(Y, \xi)$ coincide with these moments only if we take into account the main exponential behavior at $\gamma=1 / 2$. As we have seen above, for large $n$, the value of the saddle point for $\nu$ [see Eq. (62)] indeed approaching zero.

Hence, we infer that the QCD parton cascade in DIS leads to the multiplicity distribution of Eq. (34) with $N=x G(Q, x)$ at $x \rightarrow 0$, as is expected from the parton model of Sec. IIC, but $x G$ should satisfy the BFKL evolution equation, and the accuracy of Eq. (34) is not very precise at small $n$.

\section{MULTIPLICITY DISTRIBUTION IN CGC APPROACH}

In Ref. [17], the density matrix is calculated in the CGC approach, using the CGC wave function from Refs. [49,50]. In the CGC approach, the large fraction of momentum is carried by the valence quarks and gluons. These fast partons emit low energy gluons whose lifetime is much shorter than the valence partons. In other words, the valence ("hard") partons can be treated as static sources of the soft gluons. The wave function of such a system of partons can be written in the form

$$
|\psi\rangle=|v\rangle \otimes|s\rangle
$$

where $|v\rangle$ characterizes the valence degrees of freedom, while $|s\rangle$ denotes the wave function of the soft gluon in the presence of the valence partons. Sign $\otimes$ does not denote a direct product, since the wave function of a soft gluon depends on the valence degrees of freedom. Using that

$|s\rangle=\mathcal{C}|0\rangle \quad$ with $\quad \mathcal{C}=\exp \left(2 i t r \int \frac{d^{2} k}{(2 \pi)^{2}} b^{i}(k) \phi_{i}^{a}(k)\right)$,

where $\phi_{i}(k) \equiv a_{i}^{+}+a(-k)$ and $b_{a}^{i}=g \rho_{a}(k) \frac{i \boldsymbol{k}_{i}}{k^{2}}+\ldots\left(\rho^{a}\right.$ is the charge density of the valence partons), and McLerranVenugopalan (MV) model for wave function $|v\rangle$, the density matrix

$$
\hat{\rho}=|v\rangle \otimes|s\rangle\langle s| \otimes\langle v|
$$

is calculated in Ref. [17]. The result of these calculation is

$$
\begin{aligned}
& \left\langle l_{c}(q), m_{c}(-q)|\hat{\rho}| \alpha_{c}(q), \beta_{c}(-q)\right\rangle \\
& \quad=(1-R) \frac{(l+\beta) !}{\sqrt{l ! m ! \alpha ! \beta !}}\left(\frac{R}{2}\right)^{l+\beta} \delta_{l+\beta, m+\alpha},
\end{aligned}
$$

with 


$$
R=\left(1+\frac{q^{2}}{2 g^{2} \mu^{2}}\right)^{-1}
$$

where $g$ is QCD coupling constant and $\mu^{2}$ determines the color charge density in the valence wave function in the MV model [25].

For the multiplicity distribution, we only need the diagonal elements of the density matrix with $l=\alpha$ and $m=\beta$, and the multiplicity is $n=l+m$. Plugging these $l$ and $m$ into Eq. (70), we obtain for the multiplicity distribution

$\frac{\sigma_{n}}{\sigma_{\text {in }}}=(1-R) \sum_{m} \frac{n !}{m !(n-m) !}\left(\frac{R}{2}\right)^{n}=(1-R) R^{n}$.

Calculating average $n=N$, we obtain

$$
N=(1-R)^{-1},
$$

and the multiplicity distribution can be rewritten in the form

$$
\frac{\sigma_{n}}{\sigma_{\text {in }}}=\frac{1}{N}\left(\frac{N-1}{N}\right)^{n}=\frac{1}{\bar{N}}\left(\frac{\bar{N}}{\bar{N}+1}\right)^{n} .
$$

We stress that Eq. (74) coincides with Eq. (34), which we derived for the QCD parton cascade.

\section{MULTIPLICITY DISTRIBUTION IN HADRON-HADRON SCATTERING}

\section{A. Interaction of two dipoles at high energies}

We first consider the high energy interactions of two dipoles with sizes $r$ and $R$ and with $r \sim R$. In Ref. [44], it is shown that in the limited range of rapidities, which is given by Eq. (1), we can safely apply the Muller, Patel, Salam, and Iancu approach for this scattering $[36,46]$ [see
Fig. 3(a)]. The scattering amplitude in this approach can be written in the form [41]

$$
\begin{aligned}
N(Y, r, R, b)= & -\sum_{n=1}^{\infty}(-1)^{n} \int \rho_{n}^{t}\left(\boldsymbol{r}_{1}, \boldsymbol{b}_{1}^{\prime}, \ldots, \boldsymbol{r}_{n}, \boldsymbol{b}_{n}^{\prime} ; \frac{1}{2} Y\right) \\
& \times \rho_{n}^{p}\left(\boldsymbol{r}_{1}^{\prime}, \boldsymbol{b}-\boldsymbol{b}_{1}^{\prime}-\boldsymbol{b}_{1}^{\prime \prime}, \ldots, \boldsymbol{r}_{n}, \boldsymbol{b}-\boldsymbol{b}_{n}^{\prime}-\boldsymbol{b}_{n}^{\prime \prime} ;-\frac{1}{2} Y\right) \\
& \times \prod_{i=1}^{n} d^{2} r_{i} \prod_{j=1}^{n} d^{2} r_{j}^{\prime} d^{2} b_{j}^{\prime} d^{2} b_{j}^{\prime \prime} N^{\mathrm{BA}}\left(r_{i}, r_{i}^{\prime}, b_{i}^{\prime \prime}\right),
\end{aligned}
$$

where $\rho_{n}^{t}$ and $\rho_{n}^{p}$ are the parton densities in the target and projectile, respectively. These densities can be calculated from $P_{n}$ using Eq. (45). $N^{\mathrm{BA}}$ is the scattering amplitude of two dipoles in the Born approximation of perturbative QCD (see Fig. 3). Eq. (75) simply states that we can consider the QCD parton cascade of Eq. (4) generated by the dipole of the size $r$ for the c.m.f. (center mass frame) rapidities from 0 to $\frac{1}{2} Y$ and the same cascade for the dipole of the size $R$ for the rapidities from 0 to $-\frac{1}{2} Y$.

Generally speaking, for the dense system of partons at $Y=0, n$ dipoles from upper cascade could interact with $m$ dipoles from the low cascade, with the amplitude $N_{n}^{m}\left(\left\{r_{i}\right\},\left\{r_{j}^{\prime}\right\}\right)$ [41]. In Eq. (75), we assume that the system of dipoles that has been created at $Y=0$ is not very dense. In this case,

$$
N_{n}^{m}\left(\left\{r_{i}\right\},\left\{r_{j}^{\prime}\right\}\right)=\delta_{n, m} \prod_{j=1}^{n}(-1)^{n-1} N^{\mathrm{BA}}\left(r_{i}, r_{i}^{\prime}, b_{i}^{\prime \prime}\right),
$$

and after integration over $\left\{r_{1}\right\}$ and $\left\{r_{j}^{\prime}\right\}$, the scattering amplitude can be reduced to a system of enhanced BFKL Pomeron diagrams, which are shown in Fig. 3(b).

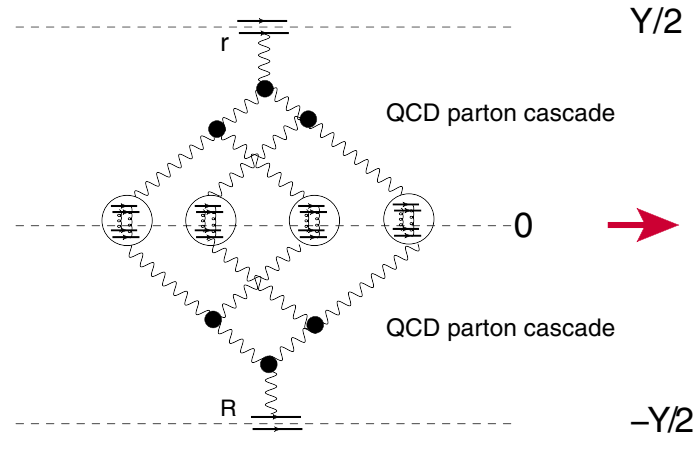

(a)

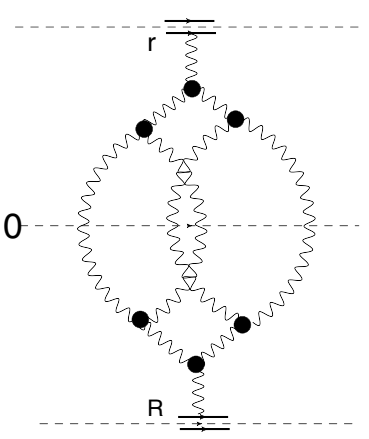

(b)

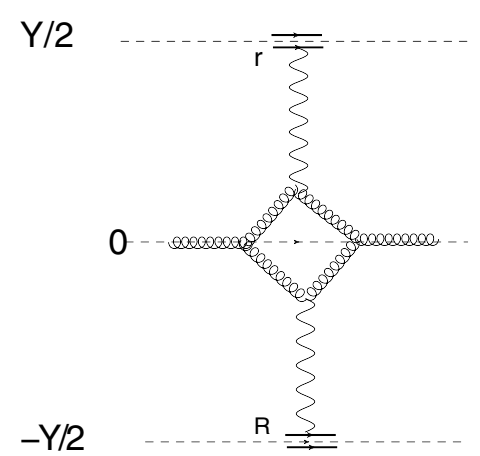

(c)

FIG. 3. Scattering amplitude for the interaction of two dipoles with sizes: $r$ and $R$ at high energy in MPSI approach [see Figs. 3(a) and $3(\mathrm{~b})]$. The amplitudes of interaction of two dipoles in the Born approximation of perturbative QCD $\left[N\left(r_{i}, r_{i}^{\prime}, b^{\prime \prime}{ }_{i}\right)\right.$ in Eq. $\left.(75)\right]$ are shown as white circles. The wavy lines denote the BFKL Pomerons. Fig. 3(c) shows the Mueller diagram [51] for inclusive production of gluons. 


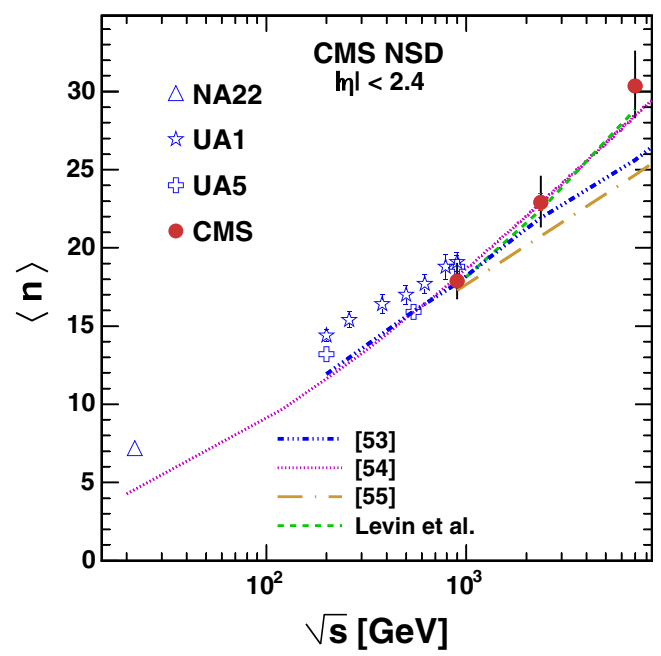

(a)

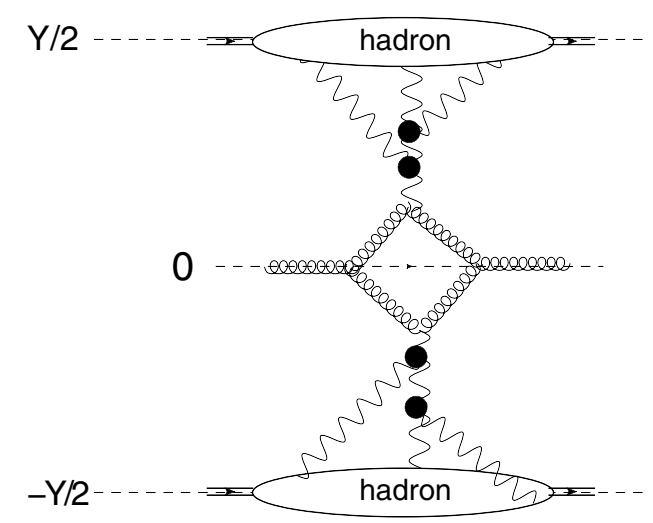

(b)

FIG. 4. (a) The comparison of the average multiplicities in proton-proton collisions at $\mid \eta \leq 2.4$ [56] with the theoretical prediction [53-55,57]. The figure is taken from Ref. [56]. The CGC prediction is marked by Levin et al., and they are taken from Ref. [57]. (b) The Mueller diagram [51] for the inclusive production in CGC/saturation approach. The wavy lines are the BFKL Pomerons. The helical lines denote the gluons. The black blobs stand for the triple Pomeron vertices.

The average number of dipoles at $Y=0$ are determined by the inclusive cross section, which is given by the diagram of Fig. 3(c) and which can be written at $y \rightarrow 0$ as follows [52]:

$$
\begin{aligned}
\frac{d \sigma}{d y d^{2} p_{T}}= & \frac{2 C_{F}}{\alpha_{s}(2 \pi)^{4}} \frac{1}{p_{T}^{2}} \int d^{2} \boldsymbol{r}_{T} e^{i \boldsymbol{p}_{T} \cdot \boldsymbol{r}_{T}} \\
& \times \int d^{2} b \nabla_{T}^{2} N^{\mathrm{BFKL}}\left(\frac{1}{2} Y ; r, r_{T} ; b\right) \\
& \times \int d^{2} B \nabla_{T}^{2} N^{\mathrm{BFKL}}\left(y_{2}=-\frac{1}{2} Y ; R, r_{T} ; B\right) .
\end{aligned}
$$

The average number of dipoles that enters the multiplicity distribution of Eq. (34) is equal to $\bar{n}=N=\int \frac{d^{2} p_{T}}{(2 \pi)^{2}} \frac{d \sigma}{d y d^{2} p_{T}} / \sigma_{\text {in }} \propto$ $\exp \left(\Delta_{\mathrm{BFKL}} Y\right)^{3}$ only if we assume that $\sigma_{\text {in }} \sim$ Const. Indeed, the enhanced diagrams of Fig. 3(b) lead to the inelastic cross section, which is constant at high energy.

\section{B. Hadron-hadron collisions}

The first idea is to view a hadron as a dilute system of dipoles and use Eq. (77) for the average multiplicity, together with the multiplicity distribution of Eq. (34). However, the energy dependence of the mean multiplicity from Eq. (77) $\left(\bar{n} \propto \exp \left(\Delta_{\mathrm{BFKL}} Y\right)\right)$ does not describe the experimental data (see Fig. 4). The experimental dependence of the mean multiplicity on energy can be parametrized as $\bar{n} \propto \exp (\lambda Y)$, but with the value of $\lambda=0.1 \div 0.2$ [53-55], which is far too small for $\Delta_{\mathrm{BFKL}}$. However, this power is close to the experimental behavior

\footnotetext{
${ }^{3} \Delta_{\mathrm{BFKL}}$ is the intercept of the BFKL Pomeron.
}

of the deep inelastic structure function. Therefore, the main assumption of Ref. [5], that $N \sim x G\left(x, Q^{2}\right)$, does not contradict the experimental data at least on the qualitative level [16].

On the other hand, the experimental data can be described in the framework of the CGC/saturation approach in which $N^{\mathrm{BFKL}}$ were replaced by $N^{\mathrm{BK}}$ [57]. Hence, we cannot view hadrons as the dilute system of dipoles but rather have to consider them as the a dense system of dipoles. For such a situation, we expect that $\bar{n} \propto Q_{S}^{2}(Y) / \bar{\alpha}_{S}$ (see Refs. [24,57-60]). Therefore, the entanglement entropy in this case is

$$
S_{\text {parton cascade }}=\ln \bar{n}=\ln \left(Q_{s}^{2}(Y) / Q_{s}^{2}(Y=0)\right) .
$$

Note that for CGC approach of Sec. III the average multiplicity turns out to be proportional, $\mu^{2} \sim Q_{s}^{2}$, if the energy evolution is taken into account (see Refs. [24,30] for review). Frankly speaking, we do not have a theoretical tool to treat the dense-dense system scattering. For a general set of diagrams [see Fig. 5(a)], we cannot use the Hamiltotian of Ref. [44] nor other theoretical methods. Therefore, our suggestion to use the multiplicity distribution of Eq. (34) with $\bar{n}$ determined by Eq. (77) with $N^{\mathrm{BK}}$ is a conjecture. However, we can claim that $\rho_{2}=\rho_{1}\left(r_{1}\right) \rho_{1}\left(r_{2}\right)-$ $\rho_{1}\left(\boldsymbol{r}_{1}+\boldsymbol{r}_{2}\right)$ [see Figs. 5(b) and 5(c)), on the same theoretical grounds as the derivation of BK equation [27,28], since the fact that the amplitude for two BFKL Pomeron production is equal to $\left(N^{\mathrm{BK}}\right)^{2}$ is used in the derivation of the BKequations.

\section{Comparison with the experimental data}

To compare the parton cascade with the experimental data, we first need to establish a relation between the 


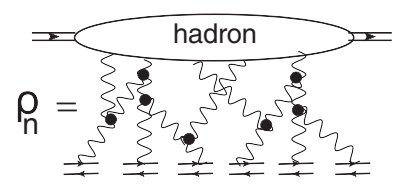

(a)

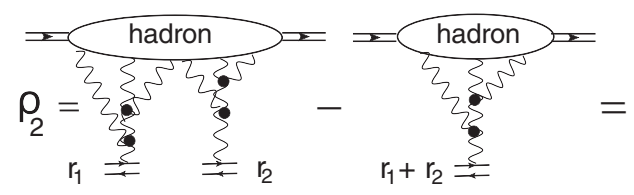

(b)

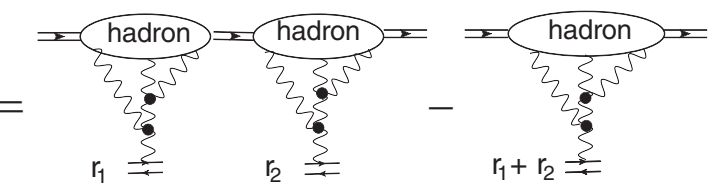

(c)

FIG. 5. (a) The BFKL Pomeron diagrams for $\rho_{n}$. (b,c) The Pomeron diagrams for $\rho_{2}$. The wavy lines denote the BFKL Pomerons, The black circles stand for triple Pomeron vertices. The arrows describe quarks and antiquarks.

multiplicity of hadrons with the multiplicities of partons, in the QCD parton cascade. Based on "parton liberation" picture [61] and on the "local parton-hadron duality" [62], we assume that $S_{\text {parton cascade }}=S_{\text {hadrons. }}$. In other words, we suggest that there is no substantial entropy increase during the transformation of partons to hadrons. This relation is our hypothesis about confinement of quarks and gluons, and it has support in the fact that the value of the entropy corresponds to a maximally entangled, equipartitioned state at a relatively modest average multiplicity of around $N=3 \div 6$.

Hence, we use Eq. (34) for the hadron multiplicity distribution replacing $\bar{n}=N$ of partons, by $\bar{n}$ of hadrons.

Following Ref. [5], we estimate, using Eq. (34), the value of the cumulants

$$
C_{q}=\frac{\left\langle n^{q}\right\rangle}{\langle n\rangle^{q}}
$$

where $\langle\ldots\rangle$ denotes the average over the distribution in hadron multiplicity $n$. These quantities can be readily computed using Eq. (34) (see Ref. [5]). The results of these estimates are the following:

$$
\begin{aligned}
& C_{2}=2-1 / \bar{n} ; \quad C_{3}=\frac{6(\bar{n}-1) \bar{n}+1}{\bar{n}^{2}} ; \\
& C_{4}=\frac{(12 \bar{n}(\bar{n}-1)+1)(2 \bar{n}-1)}{\bar{n}^{3}} ; \\
& C_{5}=\frac{(\bar{n}-1)\left(120 \bar{n}^{2}(\bar{n}-1)+30 \bar{n}\right)+1}{\bar{n}^{4}} .
\end{aligned}
$$

Using the experimental multiplicity in the rapidity window $|\eta| \leq 0.5$ equal [56] to $\bar{n}=6.33 \pm 0.46$ at $W=$ $7 \mathrm{TeV}$ and $\bar{n}=3.72 \pm 0.23$ at $W=0.9 \mathrm{TeV}$, we get from Eq. (80) the following predictions for the cumulants: $C_{2} \simeq 1.83(1.73), C_{3} \simeq 5.08(4.46), C_{4} \simeq 18.6(15.31)$, and $C_{5} \simeq 85.7(65.75)$. We put in parentheses the values at $W=0.9 \mathrm{TeV}$. The CMS experiments give (see Fig. 6(b) of Ref. [56]) $C_{2}^{\exp }=2.0 \pm 0.05, \quad C_{3}^{\exp }=5.9 \pm 0.6$, $C_{4}^{\text {exp }}=21 \pm 2$, and $C_{5}^{\exp }=90 \pm 19$. Therefore, our estimates are in reasonably good agreement with the data, indicating that the parton distributions are close to the hadronic ones. Taking the limit of $\bar{n} \rightarrow \infty$, we obtain the maximal values for the cumulants $C_{2}=2, C_{3}=6$, $C_{4}=24$, and $C_{5}=120$ as a prediction for asymptotically high energies. Comparing these numbers to the experimental values listed above, we see that the multiplicity distribution measured at $\sqrt{s}=7 \mathrm{TeV}$ is already quite close to the expected asymptotic form.

In Figs. 6 and 7, we plot the multiplicity dependence in the form of the KNO (Koba-Nielsen-Olesen) scaling function [63]:

$$
\frac{\sigma_{n}}{\sigma_{\text {in }}}=\frac{1}{\bar{n}} \Psi\left(z=\frac{n}{\bar{n}}\right)
$$

One can see that at large $\bar{n}$ the distribution of Eq. (34) leads to

$$
\Psi\left(z=\frac{n}{\bar{n}}\right) \stackrel{\bar{n} \gg 1}{\longrightarrow} e^{-z}\left(1+\frac{1}{\bar{n}}-\frac{z}{2 \bar{n}}\right)
$$

and shows KNO scaling for $\bar{n} \gg z$. As far as we know, this is the first time that $\mathrm{KNO}$ scaling appears at ultrahigh energies on theoretical grounds for hadron-hadron collisions. At least, in the framework of the Pomeron calculus [64], KNO scaling is expected only for the intermediate range of energy $[65,66]$.

In Fig. 6(a) and Fig. 7(a), we compare the CMS data [56] with the multiplicity distribution of Eq. (34). In spite of the good agreement at small $z$, one can see two major qualitative disagreements: (i) KNO scaling works better for $|\eta|<0.5$ than at $|\eta|<2.4$ in the data, while Eq. (34) predicts a different behavior, and (ii) Eq. (34) predicts a larger cross section in the region of large $z$ than is observed experimentally. The first disagreement is intimately related to the small value of $\bar{n}$ at $|\eta|<0.5$. In the framework of our approach, the violation of $\mathrm{KNO}$ scaling will not be seen if we take $N=5.8$ [67] at $\eta=0$, instead of $6.33=30.4 / 4.8$, which we used in these figures. The second disagreement is of a principle nature. As we have discussed, we use Eq. (76), which is based on the assumption that we do not have a dense system of parton at $Y=0$. Certainly, such an assumption is not correct for events with large multiplicities. Therefore, this disagreement can be considered as an additional argument in the attempts to build a generalization of CGC approach to describe the hadron-hadron collisions.

In Figs. 6(b) and 7(b), we use the negative binomial distribution of Eq. (35) in which we fixed the parameter $r$ 


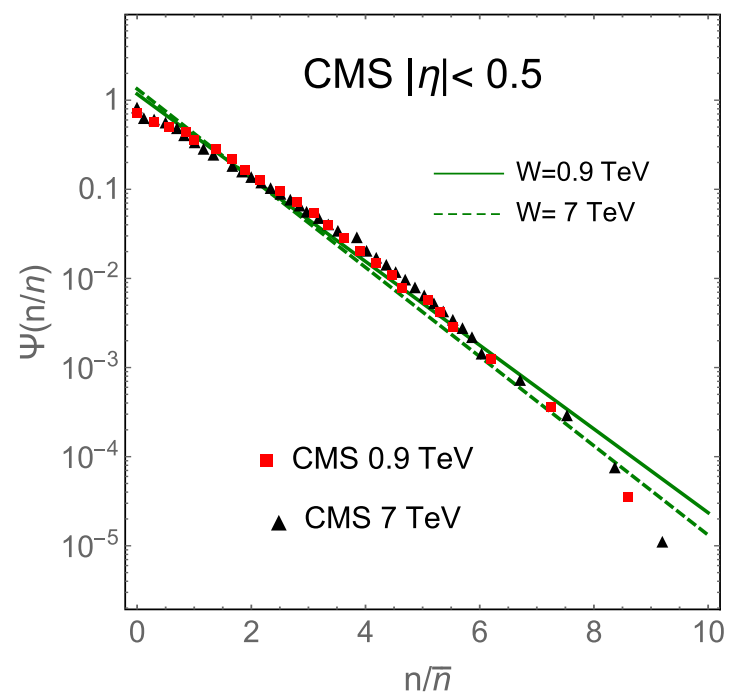

(a)

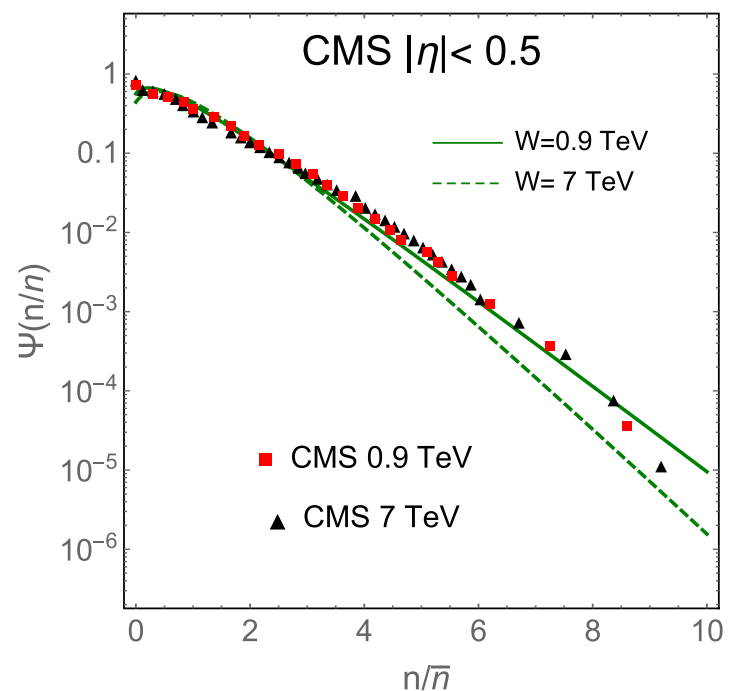

(b)

FIG. 6. KNO function $\left(\frac{\sigma_{n}}{\sigma_{\text {in }}}=\frac{1}{\bar{n}} \Psi\left(\frac{n}{\bar{n}}\right)\right)$ vs $z=n / \bar{n}$ for rapidity window $|\eta|<0.5$. (a) Comparison with prediction of Eq. (34). (b) Comparison with the negative binomial distribution of Eq. (35), in which we estimated parameter $r$ using $\rho_{2}=$ $\rho_{1}\left(r_{1}\right) \rho_{1}\left(r_{2}\right)-\rho_{1}\left(\boldsymbol{r}_{1}+\boldsymbol{r}_{2}\right)$.

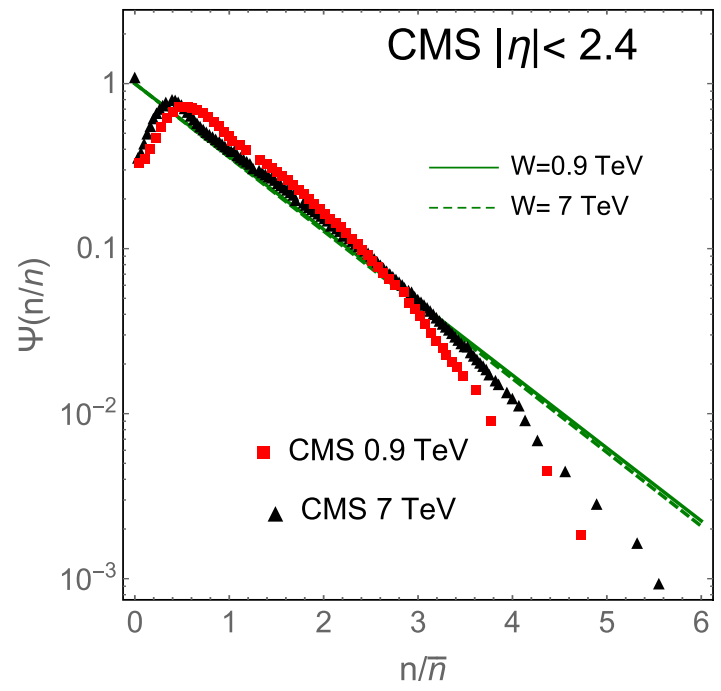

(a)

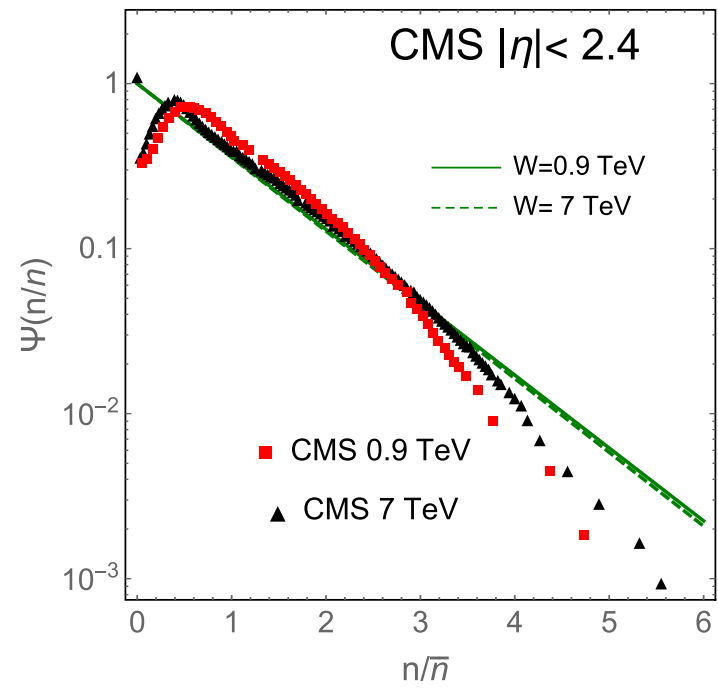

(b)

FIG. 7. KNO function $\left(\frac{\sigma_{n}}{\sigma_{i n}}=\frac{1}{\bar{n}} \Psi\left(\frac{n}{\bar{n}}\right)\right)$ vs $z=n / \bar{n}$ for rapidity window $|\eta|<2.4$. (a) Comparison with prediction of Eq. (34). (b) Comparison with the negative binomial distribution of Eq. (35), in which we estimated parameter $r$ using $\rho_{2}=\rho_{1}\left(r_{1}\right) \rho_{1}\left(r_{2}\right)-\rho_{1}\left(\boldsymbol{r}_{1}+\boldsymbol{r}_{2}\right)$.

from the moment $\left\langle\frac{n(n-1)}{2}\right\rangle$, which is estimated using $\rho_{2}=\rho_{1}\left(r_{1}\right) \rho_{1}\left(r_{2}\right)-\rho_{1}\left(\boldsymbol{r}_{1}+\boldsymbol{r}_{2}\right)$ (see Fig. 5). One can see that this distribution has the same characteristic features as Eq. (34).

\section{Back to QCD motivated parton model}

In the previous section, we inferred that comparison with the experimental data indicates that we cannot use
Eq. (76), which stems from the assumption that the system of partons, which is produced at $Y=0$, is rather dilute. Unfortunately, we have not developed a theoretical approach in the framework of QCD to treat this problem. However, in the QCD parton model, that we have discussed above, a breakthrough has been made by Ref. [47], and an approach has been constructed that sums all Pomeron diagrams of the most general type (see Figs. 3 and 5 for examples). 
In Ref. [47], a new Hamiltonian is suggested and has the form

$$
\mathcal{H}_{\mathrm{PM}}=-\frac{1}{\gamma} \bar{P} P
$$

where PM stands for "parton model." $\bar{P}$ and $P$ are the BFKL Pomeron field in the model, where the sizes of the dipoles are fixed. This Hamiltonian in the limit of small $\bar{P}$ reproduces the BK Hamiltonian (see Ref. [47] and below for details). This condition is the most important one for fixing the form of $\mathcal{H}_{\mathrm{PM}}$. The second of such conditions is that this Hamiltonian satisfies both $t$ and $s$ channel unitarity. $\gamma$ in Eq. (83) has the physical meaning of the dipole-dipole scattering amplitude in the Born approximation of perturbative QCD, and, being such, it is naturally small and of order $\bar{\alpha}_{S}$.

The most important ingredient of this approach is the generalization of the commutation relation, which has the form

$$
(1-P)(1-\bar{P})=(1-\gamma)(1-\bar{P})(1-P) .
$$

Eq. (84) gives the correct factor $(1-\gamma)^{\bar{n}}$ that includes all multiple scattering corrections, while all the dipoles remain intact and can subsequently scatter on an additional projectile or on target dipoles. For small $\gamma$, and in the regime where $P$ and $\bar{P}$ are small themselves, we obtain

$$
[P, \bar{P}]=-\gamma+\cdots,
$$

consistent with our original expression. One can see that these commutation relations take into account the interaction of one dipole with many other partons, and therefore we are going beyond the approximation, which is given by Eq. (76). Concluding this brief outline of this approach, we see that for the first time we have a simple theory in which we can describe the interactions of dilute-dilute parton system scattering as well as dilute-dense and dense-dense system interactions.

For $\mathcal{H}_{\mathrm{PM}}$, the cascade equation takes the form (see Eq. (5.8) of Ref. [47])

$$
\begin{aligned}
\frac{d P_{n}(Y)}{d Y}= & -\frac{\Delta}{\gamma}\left(1-(1-\gamma)^{n}\right) P_{n}(Y) \\
& +\frac{\Delta}{\gamma}\left(1-(1-\gamma)^{n-1}\right) P_{n-1}(Y) .
\end{aligned}
$$

For small $n(\gamma n<1)$, one can see, that Eq. (86) reduces to Eq. (39). Hence, for such small $n$, we have the multiplicity distribution of Eq. (41) with $\langle n\rangle=e^{\Delta Y}$. However, at large $n$, Eq. (86) has the form

$$
\frac{d P_{n}(Y)}{d Y}=-\frac{\Delta}{\gamma} P_{n}(Y)+\frac{\Delta}{\gamma} P_{n-1}(Y) .
$$

We will show below that this equation gives the Poisson distribution with $\langle n\rangle=\frac{\Delta}{\gamma} Y$. Therefore, as we have guessed, the interaction of one dipole with many dipoles at $Y=0$ in Fig. 3 would lead to far fewer multiplicities than Eq. (41). Using Laplace transform of Eq. (27) and following the pattern described in Sec. IIC, we obtain the solution in $\omega$-representation,

$$
P_{n}(\omega)=\frac{1}{\omega_{1}} \prod_{m=1}^{n} \frac{\omega_{m}}{\omega+\omega_{m}},
$$

where $\omega_{m}=\frac{\Delta}{\gamma}\left(1-(1-\gamma)^{m}\right)$.

We have not found an elegant form for the inverse Laplace transform, but we can see the main qualitative features of this solution, assuming that for $n<n_{0}$ with $\gamma n_{0} \approx 1$ we have $\omega_{m}=m \Delta$, but for $n>n_{0} \omega_{m}=\frac{\Delta}{\gamma}$. In this approach, we obtain

$$
\begin{aligned}
n< & n_{0} P_{n}(Y)=e^{-\Delta Y}\left(1-e^{-\Delta Y}\right)^{n-1} ; \\
n>n_{0} P_{n}(Y)= & \int^{Y} d Y^{\prime} e^{-\Delta\left(Y-Y^{\prime}\right)}\left(1-e^{-\Delta\left(Y-Y^{\prime}\right)}\right)^{n_{0}-1} \\
& \times \underbrace{e^{-\frac{\Delta}{\gamma} Y^{\prime}} \frac{\left(\frac{\Delta}{\gamma} Y^{\prime}\right)^{n-n_{0}}}{\left(n-n_{0}\right) !}}_{\text {Poisson distribution }} .
\end{aligned}
$$

The Poisson distribution in Eq. (89b) is the inverse Laplace transform of

$$
\frac{\left(\frac{\Delta}{\gamma}\right)^{n-n_{0}}}{\left(\omega+\frac{\Delta}{\gamma}\right)^{n-n_{0}+1}} .
$$

In Fig. 8, we compare this multiplicity distribution with Eq. (34). One can see that at large multiplicities the modified distributions of Eq. (89a) and Eq. (89b) lead to the suppression of the parton emission, as we expected. Of course, this modified distribution is very approximate and can only be used to clarify the qualitative features of the interaction of the partons in the exact approach.

To illustrate that the parton cascade of Eq. (86) is able to describe the experimental data, we calculate the first two $P_{1}$ and $P_{2}$, taking integral over $\omega$ in Eq. (27),

$$
\begin{aligned}
& P_{1}(Y)=e^{\Delta Y} ; \\
& P_{2}(Y)=\frac{\omega_{2}}{\omega_{2}-\omega_{1}} e^{-\omega_{1} Y}\left(1-e^{-\left(\omega_{2}-\omega_{1}\right) Y}\right),
\end{aligned}
$$

where $\omega_{2}-\omega_{1}=\Delta-\gamma \Delta<\Delta . P_{2}$ in our notation with $N=e^{\Delta Y}$ can be r-written in the form

$$
P_{2}=\frac{1}{N}\left(1-\left(\frac{1}{N}\right)^{1-\gamma}\right)
$$




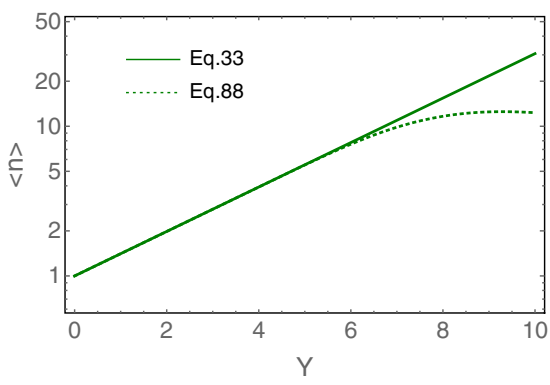

(a)

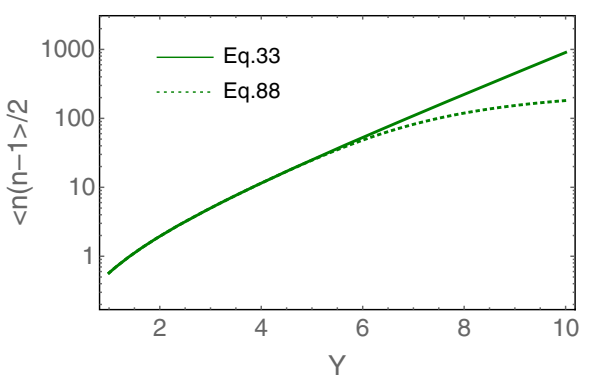

(b)

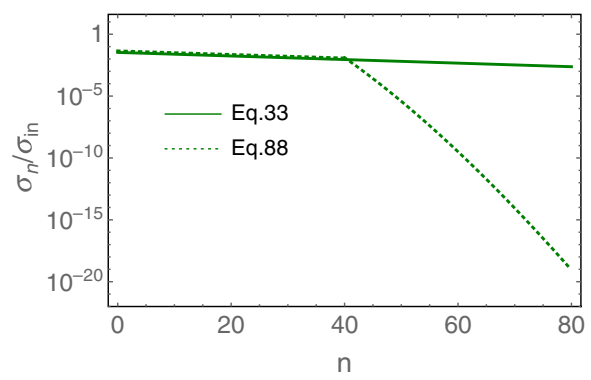

(c)

FIG. 8. Comparison of the multiplicity distribution, given by Eq. (34) with the modified distribution of Eq. (89a) and Eq. (89b).

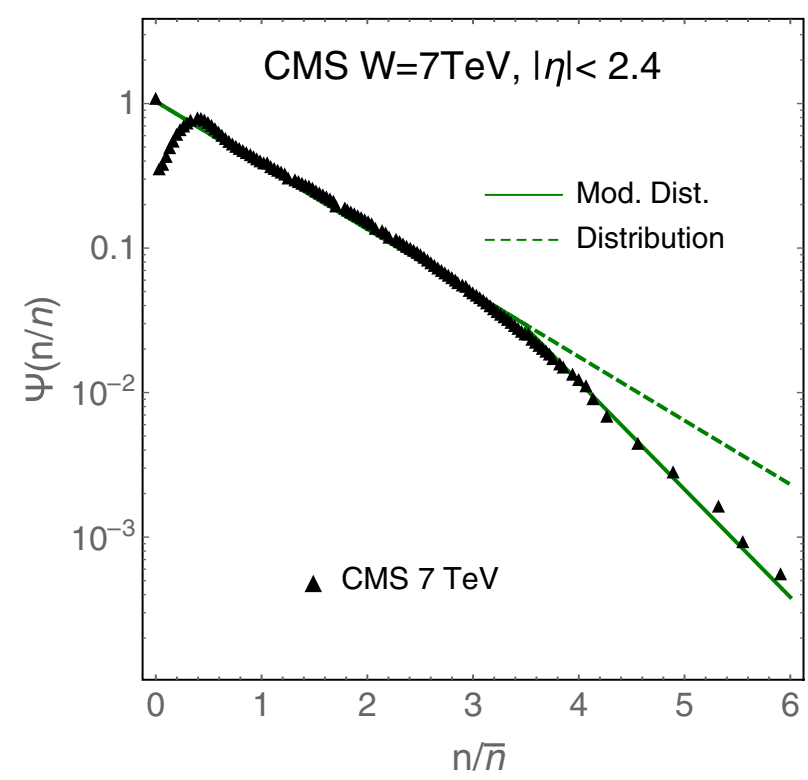

FIG. 9. KNO function $\left(\frac{\sigma_{n}}{\sigma_{\text {in }}}=\frac{1}{\bar{n}} \Psi\left(\frac{n}{\bar{n}}\right)\right)$ vs $z=n / \bar{n}$ for rapidity window $|\eta|<2$.4. Comparison between multiplicity distribution of Eq. (34) and modified distribution of Eq. (93), which was used for $z>3.5$. $\gamma$ is equal to 0.15 . The data are taken from Ref. [56].

Assuming that the multiplicity distribution has the form

$$
P_{n}=\frac{1}{N}\left(1-\left(\frac{1}{N}\right)^{1-\gamma}\right)^{n-1}
$$

we can use this approximation, except for very large $n$. In Fig. 9, we compare Eq. (93) with the data. One can see that it provides quite a good description of the data.

\section{CONCLUSIONS}

As has been discussed in the Introduction, this paper has two main results. First, we prove that in QCD at high energies the multiplicity distribution has the form of Eq. (34), which was discussed in Ref. [5], in the framework of the parton model. We also show that the average number of gluons is not always related to the gluon structure function and can depend on both energy and the size of the dipoles. However, the entanglement entropy is equal to $S_{\text {partons }}=\ln N$, where $N$ is the average number of partons, confirming that the partonic state at high energy is maximally entangled [5]. In the case of DIS, we prove that the average number of partons is related to the gluon structure function, and only if we use the BFKL evolution equation for this structure function can we prove that the multiplicity distribution has the form of Eq. (34).

Second, we developed an approach for hadronhadron collisions in which we show that Eq. (34) correctly describes the Mueller-Patel-Salam-Iancu approach $[36,46]$. We argued that actually at high energies the mean multiplicity is proportional to $Q_{s}^{2}(Y)$, leading to the entanglement entropy proportional to $\ln Q_{s}^{2}(Y)$.

We compared the multiplicity distribution with the experimental data. We described quite well the data at multiplicities $n<(3 \div 4)\langle n\rangle$, using Eq. (34), but predict higher $\sigma_{n}$ for large multiplicities than have been seen experimentally. We conclude that this indicates that the assumptions that at high energies we create a dilute system of partons in the c.m. rapidity $Y=0$, and we can use Eq. (76), are not correct, and we have to deal with a rather dense system of partons. At the moment, we have not developed theoretical tools to treat such a system. However, in Ref. [47], an approach was developed for the parton model, which allow us to theoretically treat such a dense system of partons. We show that in this approach the production of a system of partons with large multiplicities is suppressed in comparison with Eq. (34), and we are able to describe the experimental data.

\section{ACKNOWLEDGMENTS}

We thank our colleagues at Tel Aviv university and Universidad T'ecnica Federico Maria (UTFSM) for encouraging discussions. This research was supported by ANID PIA/APOYO AFB180002 (Chile) and Fondecyt (Chile) Grant No. 1180118. 
[1] K. Kutak, Gluon saturation and entropy production in proton-proton collisions, Phys. Lett. B 705, 217 (2011).

[2] R. Peschanski, Dynamical entropy of dense QCD states, Phys. Rev. D 87, 034042 (2013).

[3] A. Kovner and M. Lublinsky, Entanglement entropy and entropy production in the color glass condensate framework, Phys. Rev. D 92, 034016 (2015).

[4] R. Peschanski and S. Seki, Entanglement entropy of scattering particles, Phys. Lett. B 758, 89 (2016).

[5] D. E. Kharzeev and E. M. Levin, Deep inelastic scattering as a probe of entanglement, Phys. Rev. D 95, 114008 (2017).

[6] O. Baker and D. Kharzeev, Thermal radiation and entanglement in proton-proton collisions at energies available at the CERN Large Hadron Collider, Phys. Rev. D 98, 054007 (2018).

[7] J. Berges, S. Floerchinger, and R. Venugopalan, Dynamics of entanglement in expanding quantum fields, J. High Energy Phys. 04 (2018) 145.

[8] Y. Hagiwara, Y. Hatta, B. W. Xiao, and F. Yuan, Classical and quantum entropy of parton distributions, Phys. Rev. D 97, 094029 (2018).

[9] N. Armesto, F. Dominguez, A. Kovner, M. Lublinsky, and V. Skokov, The color glass condensate density matrix: Lindblad evolution, entanglement entropy and Wigner functional, J. High Energy Phys. 05 (2019) 025.

[10] E. Gotsman and E. Levin, Thermal radiation and inclusive production in the $\mathrm{CGC}$ /saturation approach at high energies, Eur. Phys. J. C 79, 415 (2019).

[11] E. Gotsman and E. Levin, Thermal radiation and inclusive production in the Kharzeev-Levin-Nardi model for ion-ion collisions, Phys. Rev. D 100, 034013 (2019).

[12] A. Kovner, M. Lublinsky, and M. Serino, Entanglement entropy, entropy production and time evolution in high energy QCD, Phys. Lett. B 792, 4 (2019).

[13] D. Neill and W. J. Waalewijn, Entropy of a Jet, Phys. Rev. Lett. 123, 142001 (2019).

[14] Y. Liu and I. Zahed, Entanglement in Regge scattering using the AdS/CFT correspondence, Phys. Rev. D 100, 046005 (2019).

[15] X. Feal, C. Pajares, and R. Vazquez, Thermal behavior and entanglement in $\mathrm{Pb}-\mathrm{Pb}$ and $\mathrm{p}-\mathrm{p}$ collisions, Phys. Rev. C 99, 015205 (2019).

[16] Z. Tu, D. E. Kharzeev, and T. Ullrich, Einstein-PodolskyRosen Paradox and Quantum Entanglement at Subnucleonic Scales, Phys. Rev. Lett. 124, 062001 (2020).

[17] H. Duan, C. Akkaya, A. Kovner, and V. V. Skokov, Entanglement, partial set of measurements, and diagonality of the density matrix in the parton model, Phys. Rev. D 101, 036017 (2020).

[18] J. D. Bjorken, Asymptotic sum rules at infinite momentum, Phys. Rev. 179, 1547 (1969).

[19] R. P. Feynman, Very High-Energy Collisions of Hadrons, Phys. Rev. Lett. 23, 1415 (1969); Photon-Hadron Interactions (CBC Press, Reading, 1972).

[20] J. D. Bjorken and E. A. Paschos, Inelastic electron-proton and $\gamma$-proton scattering and the structure of the nucleon, Phys. Rev. 185, 1975 (1969).

[21] V. N. Gribov, Inelastic processes at super high-energies and the problem of nuclear cross-sections, Yad. Fiz. 9,
640 (1969) [Sov. J. Nucl. Phys. 9, 369 (1969)]; in Elementary Particles (1st ITEP School in Physics) (Atomizdat, Moscow, 1973), Issue 1, p. 65.

[22] V. N. Gribov, B. L. Ioffe, and I. Y. Pomeranchuk, What is the range of interactions at high-energies, Yad. Fiz. 2, 768 (1965) [Sov. J. Nucl. Phys. 2, 549 (1966)].

[23] B. L. Ioffe, Space-time picture of photon and neutrino scattering and electroproduction cross-section asymptotics, Phys. Lett. 30B, 123 (1969).

[24] Y. V. Kovchegov and E. Levin, Quantum Chromodynamics at High Energies, Cambridge Monographs on Particle Physics, Nuclear Physics and Cosmology (Cambridge University Press, Cambridge, England, 2012).

[25] L. McLerran and R. Venugopalan, Computing quark and gluon distribution functions for very large nuclei, Phys. Rev. D 49, 2233 (1994); Gluon distribution functions for very large nuclei at small transverse momentum, Phys. Rev. D 49, 3352 (1994); Green's function in the color field of a large nucleus, Phys. Rev. D 50, 2225 (1994); Fock space distributions, structure functions, higher twists, and small $x$, Phys. Rev. D 59, 094002 (1999).

[26] A. H. Mueller, Soft Gluons In the infinite momentum wave function and the BFKL Pomeron, Nucl. Phys. B415, 373 (1994); Unitarity and the BFKL pomeron, Nucl. Phys. B437, 107 (1995).

[27] I. Balitsky, Operator expansion for high-energy scatte ring, Nucl. Phys. B463, 99 (1996); Factorization and high-energy effective action, Phys. Rev. D 60, 014020 (1999).

[28] Y. V. Kovchegov, Small-x $F_{2}$ structure function of a nucleus including multiple Pomeron exchanges Phys. Rev. D 60, 034008 (1999).

[29] J. Jalilian-Marian, A. Kovner, A. Leonidov, and H. Weigert, The BFKL equation from the Wilson renormalization group, Nucl. Phys. B504, 415 (1997); The Wilson renormalization group for low x physics: Towards the high density regime, Phys. Rev. D 59, 014014 (1998); A. Kovner, J. G. Milhano, and $\mathrm{H}$. Weigert, Relating different approaches to nonlinear QCD evolution at finite gluon density, Phys. Rev. D 62, 114005 (2000); E. Iancu, A. Leonidov, and L. D. McLerran, Nonlinear gluon evolution in the color glass condensate. I, Nucl. Phys. A692, 583 (2001); E. Iancu, A. Leonidov, and L. D. McLerran, The renormalization group equation for the color glass condensate, Phys. Lett. B 510, 133 (2001); E. Ferreiro, E. Iancu, A. Leonidov, and L. McLerran, Nonlinear gluon evolution in the color glass condensate. II, Nucl. Phys. A703, 489 (2002); H. Weigert, Unitarity at small Bjorken $x$, Nucl. Phys. A703, 823 (2002).

[30] F. Gelis, E. Iancu, J. Jalilian-Marian, and R. Venugopalan, The color glass condensate, Annu. Rev. Nucl. Part. Sci. 60, 463 (2010).

[31] V. S. Fadin, E. A. Kuraev, and L. N. Lipatov, On the pomeranchuk singularity in asymptotically free theories, Phys. Lett. 60B, 50 (1975); E. A. Kuraev, L. N. Lipatov, and V. S. Fadin, The Pomeranchuk singularity in nonabelian gauge theories, Zh. Eksp. Teor. Fiz. 72, 377 (1977) [Sov. Phys. JETP 45, 199 (1977)]; I. I. Balitsky and L. N. Lipatov, The Pomeranchuk singularity in quantum chromodynamics, Yad. Fiz. 28, 1597 (1978) [Sov. J. Nucl. Phys. 28, 822 (1978)]. 
[32] L. N. Lipatov, The bare Pomeron in quantum chromodynamics, Zh. Eksp. Teor. Fiz. 90, 1536 (1986) [Sov. Phys. JETP 63, 904 (1986)].

[33] L. V. Gribov, E. M. Levin, and M. G. Ryskin, Semihard processes in QCD, Phys. Rep. 100, 1 (1983).

[34] E. M. Levin and M. G. Ryskin, High-energy hadron collisions in QCD, Phys. Rep. 189, 268 (1990).

[35] A. H. Mueller and J. Qiu, Gluon recombination and shadowing at small values of $x$, Nucl. Phys. B268, 427 (1986).

[36] A. H. Mueller and B. Patel, Single and double BFKL pomeron exchange and a dipole picture of high-energy hard processes, Nucl. Phys. B425, 471 (1994).

[37] J. Bartels, M. Braun, and G. Vacca, Pomeron vertices in perturbative QCD in diffractive scattering, Eur. Phys. J. C 40, 419 (2005); J. Bartels and C. Ewerz, Unitarity corrections in high-energy QCD, J. High Energy Phys. 09 (1999) 026; J. Bartels and M. Wusthoff, The triple Regge limit of diffractive dissociation in deep inelastic scattering, Z. Phys. C 66, 157 (1995); J. Bartels, Unitarity corrections to the Lipatov pomeron and the four gluon operator in deep inelastic scattering in QCD, Z. Phys. C 60, 471 (1993).

[38] M. Braun, Conformal invariant pomeron interaction in the perurbative QCD with large $N_{c}$, Phys. Lett. B 632, 297 (2006); Nucleus nucleus interaction in the perturbative QCD, Eur. Phys. J. C 33, 113 (2004); Nucleus-nucleus scattering in perturbative QCD with $N_{c} \rightarrow$ infinity, Phys. Lett. B 483, 115 (2000); Structure function of the nucleus in the perturbative QCD with $N_{c} \rightarrow$ infinity (BFKL pomeron fan diagrams), Eur. Phys. J. C 16, 337 (2000); The system of four reggeized gluons and the three-pomeron vertex in the high colour limit Eur. Phys. J. C 6, 321 (1999); M. Braun and G. Vacca, Triple pomeron vertex in the limit $N_{c} \rightarrow$ infinity, Eur. Phys. J. C 6, 147 (1999).

[39] Y. V. Kovchegov and E. Levin, Diffractive dissociation including multiple pomeron exchanges in high parton density QCD, Nucl. Phys. B577, 221 (2000).

[40] E. Levin and M. Lublinsky, Towards a symmetric approach to high energy evolution: Generating functional with Pomeron loops, Nucl. Phys. A763, 172 (2005).

[41] E. Levin and M. Lublinsky, Balitsky's hierarchy from Mueller's dipole model and more about target correlations, Phys. Lett. B 607, 131 (2005); A linear evolution for nonlinear dynamics and correlations in realistic nuclei, Nucl. Phys. A730, 191 (2004).

[42] E. Levin, J. Miller, and A. Prygarin, Summing Pomeron loops in the dipole approach, Nucl. Phys. A806, 245 (2008).

[43] T. Altinoluk, C. Contreras, A. Kovner, E. Levin, M. Lublinsky, and A. Shulkim, QCD reggeon calculus from JIMWLK evolution, Int. J. Mod. Phys. Conf. Ser. 25, 1460025 (2014); T. Altinoluk, N. Armesto, A. Kovner, E. Levin, and M. Lublinsky, KLWMIJ Reggeon field theory beyond the large $N_{c}$ limit, J. High Energy Phys. 08 (2014) 007.

[44] T. Altinoluk, A. Kovner, E. Levin, and M. Lublinsky, Reggeon field theory for large Pomeron loops, J. High Energy Phys. 04 (2014) 075; T. Altinoluk, C. Contreras, A. Kovner, E. Levin, M. Lublinsky, and A. Shulkin, QCD Reggeon calculus from KLWMIJ/JIMWLK evolution: Vertices, Reggeization and all, J. High Energy Phys. 09 (2013) 115.
[45] E. Levin, Dipole-dipole scattering in CGC/saturation approach at high energy: Summing Pomeron loops, J. High Energy Phys. 11 (2013) 039.

[46] A. H. Mueller and G. Salam, Large multiplicity fluctuations and saturation effects in onium collisions, Nucl. Phys. B475, 293 (1996); G. Salam, Studies of unitarity at small x using the dipole formulation, Nucl. Phys. B461, 512 (1996); E. Iancu and A. Mueller, Rare fluctuations and the high-energy limit of the S matrix in QCD, Nucl. Phys. A730, 494 (2004); From color glass to color dipoles in high-energy onium onium scattering, Nucl. Phys. A730, 460 (2004).

[47] A. Kovner, E. Levin, and M. Lublinsky, QCD unitarity constraints on Reggeon field theory, J. High Energy Phys. 08 (2016) 031.

[48] I. Gradstein and I. Ryzhik, Table of Integrals, Series, and Products, 5th ed. (Academic Press, London, 1994).

[49] A. Kovner, M. Lublinsky, and U. Wiedemann, From bubbles to foam: Dilute to dense evolution of hadronic wave function at high energy, J. High Energy Phys. 06 (2007) 075.

[50] T. Altinoluk, A. Kovner, M. Lublinsky, and J. Peressutti, QCD Reggeon field theory for every day: Pomeron loops included, J. High Energy Phys. 03 (2009) 109.

[51] A. H. Mueller, $\mathrm{O}(2,1)$ analysis of single particle spectra at high energy, Phys. Rev. D 2, 2963 (1970).

[52] Y. V. Kovchegov and K. Tuchin, Inclusive gluon production in DIS at high parton density, Phys. Rev. D 65, 074026 (2002).

[53] E. Gotsman, A. Kormilitzin, E. Levin, and U. Maor, QCD motivated approach to soft interactions at high energies: Nucleus-nucleus and hadron-nucleus collisions, Nucl. Phys. A842, 82 (2010).

[54] A. Likhoded, A. Luchinsky, and A. Novoselov, Light hadron production in inclusive pp-scattering at LHC, Phys. Rev. D 82, 114006 (2010).

[55] A. Kaidalov and M. Poghosyan, Predictions of quark-gluon string model for pp at LHC, Eur. Phys. J. C 67, 397 (2010).

[56] V. Khachatryan et al. (CMS Collaboration), Charged particle multiplicities in $p p$ interactions at $\sqrt{s}=0.9,2.36$, and 7 TeV, J. High Energy Phys. 01 (2011) 079.

[57] E. Levin and A. H. Rezaeian, Gluon saturation and inclusive hadron production at LHC, Phys. Rev. D 82, 014022 (2010).

[58] D. Kharzeev and E. Levin, Manifestations of high density QCD in the first RHIC data, Phys. Lett. B 523, 79 (2001); D. Kharzeev, E. Levin, and M. Nardi, The onset of classical QCD dynamics in relativistic heavy ion collisions, Phys. Rev. C 71, 054903 (2005); Hadron multiplicities at the LHC, J. Phys. G 35, 054001 (2008).

[59] A. Dumitru, D. E. Kharzeev, E. M. Levin, and Y. Nara, Gluon saturation in $p A$ collisions at the LHC: KLN model predictions for hadron multiplicities, Phys. Rev. C 85, 044920 (2012).

[60] T. Lappi, Energy dependence of the saturation scale and the charged multiplicity in pp and AA collisions, Eur. Phys. J. C 71, 1699 (2011).

[61] A. H. Mueller, Toward equilibration in the early stages after a high-energy heavy ion collision, Nucl. Phys. B572, 227 (2000). 
[62] Y. L. Dokshitzer, V. A. Khoze, S. I. Troian, and A. H. Mueller, QCD coherence in high-energy reactions, Rev. Mod. Phys. 60, 373 (1988).

[63] Z. Koba, H. B. Nielsen, and P. Olesen, Scaling of multiplicity distributions in high-energy hadron collisions, Nucl. Phys. B40, 317 (1972).

[64] V. N. Gribov, A reggeon diagram technique, Zh. Eksp. Teor. Fiz. 53, 654 (1967) [Sov. Phys. JETP 26, 414 (1967)].
[65] V. Abramovskii and O. Kancheli, Regge branching and distribution of hadron multiplicity at high energies, Pis'ma Zh. Eksp. Teor. Fiz. 15, 559 (1972).

[66] S. G. Matinyan and W. Walker, Multiplicity distribution and mechanisms of the high-energy hadron collisions, Phys. Rev. D 59, 034022 (1999), and reference therein.

[67] C. Patrignani et al. (Particle Data Group), Review of particle physics, Chin. Phys. C 40, 100001 (2016). 